University of Nebraska - Lincoln DigitalCommons@University of Nebraska - Lincoln

2017

\title{
Recent developments in accuracy and stability improvement of nonlinear filter methods for DNS and LES of compressible flows
}

Helen Yee

NASA Ames Research Center, yee@nas.nasa.gov

Björn Sjögreen

NASA Ames Research Center, Bjorn.Sjogreen@multid.se

Follow this and additional works at: http://digitalcommons.unl.edu/nasapub

Yee, Helen and Sjögreen, Björn, "Recent developments in accuracy and stability improvement of nonlinear filter methods for DNS and LES of compressible flows" (2017). NASA Publications. 250.

http://digitalcommons.unl.edu/nasapub/250

This Article is brought to you for free and open access by the National Aeronautics and Space Administration at DigitalCommons@University of Nebraska - Lincoln. It has been accepted for inclusion in NASA Publications by an authorized administrator of DigitalCommons@University of Nebraska - Lincoln. 


\title{
Recent developments in accuracy and stability improvement of nonlinear filter methods for DNS and LES of compressible flows
}

\author{
H.C. Yee*, Björn Sjögreen
}

NASA Ames Research Center, United States

\section{A R T I C L E I N F O}

Article history:

Received 3 June 2017

Revised 8 August 2017

Accepted 14 August 2017

Available online $\mathrm{xxx}$

\section{Keywords:}

High order methods

High order shock-capturing methods

Stability improvement of high order methods

Accuracy improvement for DNS \& LES

\begin{abstract}
A B S T R A C T
Recent progress in the improvement of numerical stability and accuracy of the Yee and Sjögreen [49] high order nonlinear filter schemes is described. The Yee \& Sjögreen adaptive nonlinear filter method consists of a high order non-dissipative spatial base scheme and a nonlinear filter step. The nonlinear filter step consists of a flow sensor and the dissipative portion of a high resolution nonlinear high order shock-capturing method to guide the application of the shock-capturing dissipation where needed. The nonlinear filter idea was first initiated by Yee et al. [54] using an artificial compression method (ACM) of Harten [12] as the flow sensor. The nonlinear filter step was developed to replace high order linear filters so that the same scheme can be used for long time integration of direct numerical simulations (DNS) and large eddy simulations (LES) for both shock-free turbulence and turbulence-shock waves interactions. The improvement includes four major new developments: (a) Smart flow sensors were developed to replace the global ACM flow sensor [21,22,50]. The smart flow sensor provides the locations and the estimated strength of the necessary numerical dissipation needed at these locations and leaves the rest of the flow field free of shock-capturing dissipation. (b) Skew-symmetric splittings were developed for compressible gas dynamics and magnetohydrodynamics (MHD) equations $[35,36]$ to improve numerical stability for long time integration. (c) High order entropy stable numerical fluxes were developed as the spatial base schemes for both the compressible gas dynamics and MHD [37,38]. (d) Several dispersion relation-preserving (DRP) central spatial schemes were included as spatial base schemes in the framework of our nonlinear filter method approach [40]. With these new scheme constructions the nonlinear filter schemes are applicable to a wider class of accurate and stable DNS and LES applications, including forced turbulence simulations where the time evolution of flows might start with low speed shock-free turbulence and develop into supersonic speeds with shocks. Representative test cases for both smooth flows and problems containing discontinuities for compressible flows are included.
\end{abstract}

Published by Elsevier Ltd.

\section{Introduction}

The construction of spatially stable and accurate numerical methods for long time integration of complex multiscale compressible shock free turbulent flows, turbulent flows containing discontinuities, steep gradients, and vortical flows is very different from shorter time integration of non-turbulence/non-acoustic unsteady flows and rapidly developing shock-wave interaction simulations. Standard direct numerical simulations (DNS) and large eddy simulations (LES) usually require high accuracy schemes with low dissipative and low dispersive errors in space and time. It is common

Yee-Sjogreen 2nd paper for a special issue in honor of Prof. Eleuterio F. Toro's 70th birthday, 2017.

* Corresponding author.

E-mail addresses: Helen.M.Yee@nasa.gov (H.C. Yee), bjorn.sjogreen@multid.se (B. Sjögreen).

http://dx.doi.org/10.1016/j.compfluid.2017.08.028

0045-7930/Published by Elsevier Ltd. to have numerically induced high frequency oscillations (spurious numerical artifacts) due to long time integration of non-dissipative or low-dissipative finite discretizations. A good numerical method for DNS and LES should be able to minimize these spurious oscillations while maintaining stability and accuracy during an entire long-time evolution. This paper only addresses the spatial discretization by the method-of-lines approach. Controlling low dissipative and low dispersive temporal errors is important but outside the scope of this investigation. Highly accurate appropriate temporal discretizations and, when appropriate, small time steps are assumed to be used in conjunction with the current development.

Numerical stability and accuracy considerations are an intricate balancing act for turbulence flows with discontinuities. More stable numerical methods usually contain more numerical dissipation than their higher accuracy method counterparts. Improving nonlinear stability without smearing physical turbulent fluctuations for long time integrations are conflicting requirements 


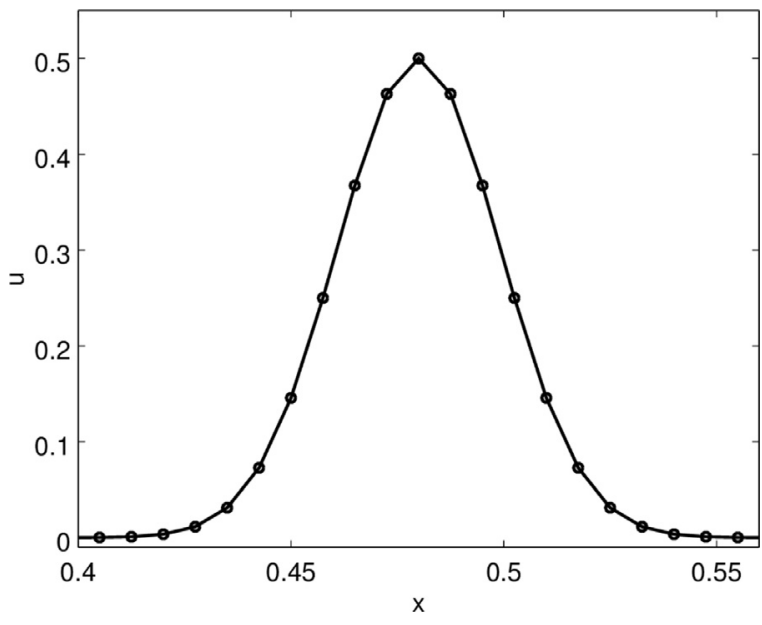

Fig. 1. Smooth initial data of the linear advection problem.

for numerical methods development. Since the turn of this century, many optimized compact and non-compact WENO schemes have been developed to address some of the pacing difficulties. See, e.g., $[10,16,27]$. These numerical methods are very high in CPU operation counts and most often still suffer from numerical stability/accuracy for long time integration. Other optimized numerical methods for combating these conflicting requirements combine the non-dissipative or low dissipative, and low dispersive spatial schemes with high order high resolution shock-capturing schemes. The blending of these two types of schemes requires extreme care to ensure numerical conservation and stability at interface locations [30]. Other more efficient numerical methods which avoid the interfacing problem are the $[21,22,33,33,49,54]$ nonlinear filter schemes. Numerical stability can be improved by skewsymmetric splitting of the inviscid flux derivatives [35,36,53] and by high order stable entropy conservative numerical fluxes [37,38]. Another source of accuracy improvement is the dispersion relationpreserving (DRP) schemes for computational aeroacoustics (CAA) [40].

Nonlinear filter schemes: The Yee and Sjögreen [50] adaptive nonlinear filter method consists of a high order non-dissipative spatial base scheme and a nonlinear filter step. The nonlinear filter step consists of a flow sensor and the dissipative portion of a high resolution high order shock-capturing method to guide the application of the shock-capturing dissipation where needed. The nonlinear filter idea was first initiated by Yee et al. [54] using an artificial compression method (ACM) of Harten [12] as the flow sensor. The nonlinear filter step was developed to replace high order linear filters so that the same scheme can be used for long time integration of direct numerical simulations (DNS) and large eddy simulations (LES) for both shock-free turbulence and turbulence-shock wave interactions. Smart flow sensors were developed at a later stage by the same investigators and collaborators in $[21,22,33,33,49]$. The smart flow sensor provides the locations and the estimated strength of the necessary numerical dissipation needed at these locations and leaves the rest of the flow field free of shock-capturing dissipations. It is noted that the nonlinear filter approach of Yee and Sjögreen [50] requires one Riemann solver per time step per grid point for each spatial direction. It is independent of the time discretization. However, hybrid schemes (switching between high order non-dissipative methods and high order shockcapturing methods) would require four Riemann solvers per time step per grid point for each spatial direction if, e.g., a fourth-stage Runge-Kutta time discretization is used. Unlike the hybrid method, our highly parallelizable adaptive nonlinear filter schemes do not rely on switching between schemes to avoid the related numerical instability and conservation consideration at switching locations. These nonlinear filter schemes with adaptive numerical dissipation control in high order shock-capturing schemes and their hybrid cousins have shown excellent performance for certain turbulent test cases. For more practical 3D test cases of DNS and LES of compressible shock-free turbulence, low speed turbulence with shocklets, and supersonic turbulence for non-periodic boundaries in curvilinear geometries, some improvement in numerical stability is needed without resorting to added numerical dissipation that can interfere with the accuracy of numerical simulations.

Skew-symmetric splitting of the inviscid flux derivative: Starting in the early 1980s skew-symmetric splitting of certain components of the inviscid flux derivatives in conjunction with central schemes was shown to help with numerical stability for long time integration. For certain splittings they can provide a stable energy norm estimate for the Euler equations with smooth flows. For other skew-symmetric formulations they can provide a discrete momentum conservation or a discrete kinetic energy preservation property. See Arakawa [1], Blaisdell et al. [2], Ducros et al. [8], Kotov et al. [21, 22], Sjögreen and Yee [34], Yee and Sjögreen [49,50], Yee et al. [53] for some discussions and performance of the combined approach for DNS and LES applications. A semiconservative skew-symmetric splitting (entropy splitting) of Yee et al. [53] in conjunction with the nonlinear filter approach to improve numerical stability without added ad hoc numerical dissipation was conducted in 2000. It has been utilized extensively in DNS of shock-free turbulence. See [32] and their later work for their wide applications. For their skew-symmetric splitting extension to the ideal magnetohydrodynamics (MHD), see Sjögreen and Yee $[35,36]$, Yee et al. [53]. Note that some of the skew-symmetric splittings for the gas dynamics flux derivatives are not applicable and/or cannot be straightforwardly extended to the ideal MHD [53]. Their degree of stability improvement is also dependent on the MHD governing equation formulation.

High order entropy conservative schemes: Entropy conservative schemes $[7,42,48]$ are another class of methods that might have better stability properties than straightforward pure centered discretizations and compact spatial schemes. Here, entropy conservative schemes refer to conservative schemes satisfying a discrete entropy equation. In view of the fact that methods proposed in $[7,42,48]$ are low order and their linear numerical dissipation approaches for shock-capturing require further improvement, Sjögreen and Yee combined some of these ideas to construct a form of the high order conservative entropy numerical fluxes. Starting with the high order entropy conservative development of Sjögreen and Yee [34] for gas dynamics in smooth flows, construction of efficient high order conservative numerical fluxes for problems containing discontinuities and for the ideal MHD are reported in Sjögreen and Yee $[37,38]$. Note that the extension of high order entropy conservative numerical fluxes that were developed for gas dynamics to the MHD is not straightforward due to the non-strictly hyperbolic nature of the conservative ideal MHD equations. See [37,38].

DRP schemes: DRP schemes (optimized low dispersion schemes) for CAA are also a class of methods that might have better accuracy than pure centered schemes. Unlike typical DNS and LES numerical considerations, the magnitude of acoustic solutions is similar to numerical noise but is different from numerically induced high frequency oscillations due to long time integration of non-dissipative or low dissipative finite discretizations. Here, the term "DRP" schemes has been used loosely, according to the recent definition of DRP methods by Tam [44], to include general schemes that perform various optimizations to reduce numerical dispersion errors for different applications. Most CAA-related DRP methods employed techniques to minimize dispersion error to resolve linear acoustic waves over long distances without compromising the 
C08

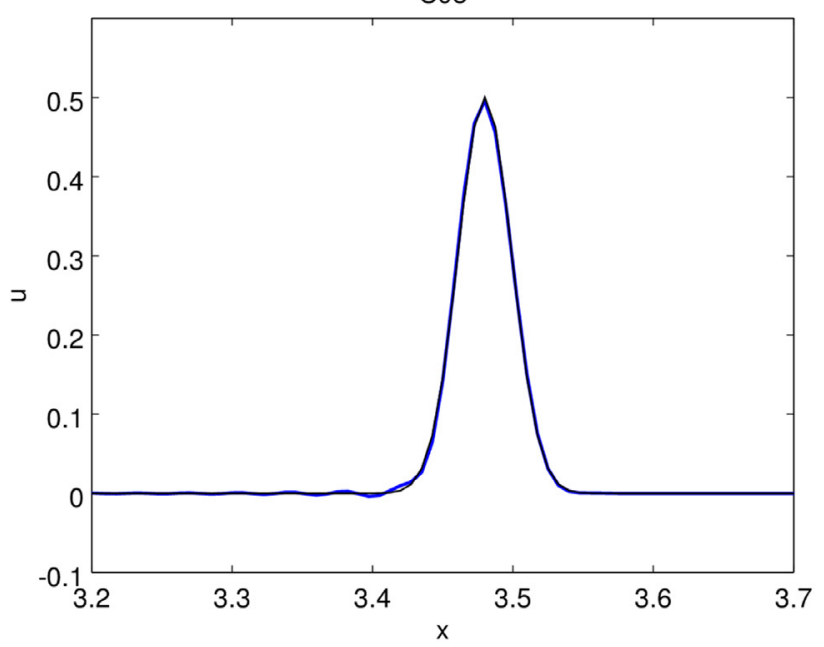

DRP4S9

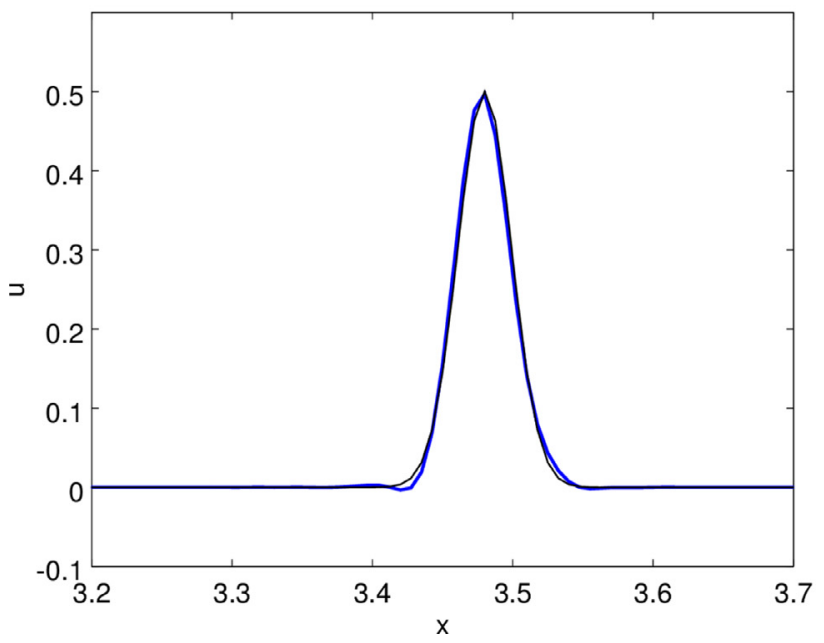

DRP4S7

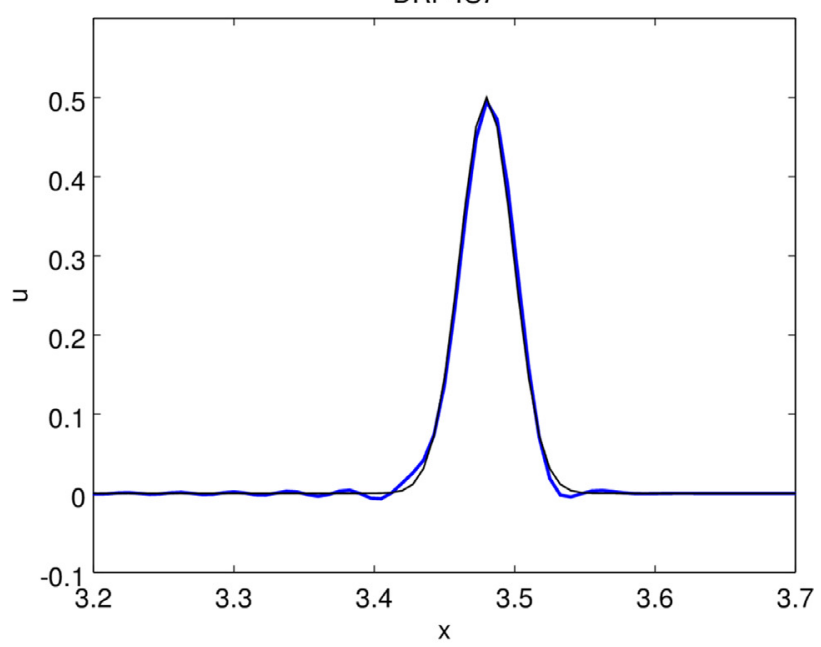

STO9

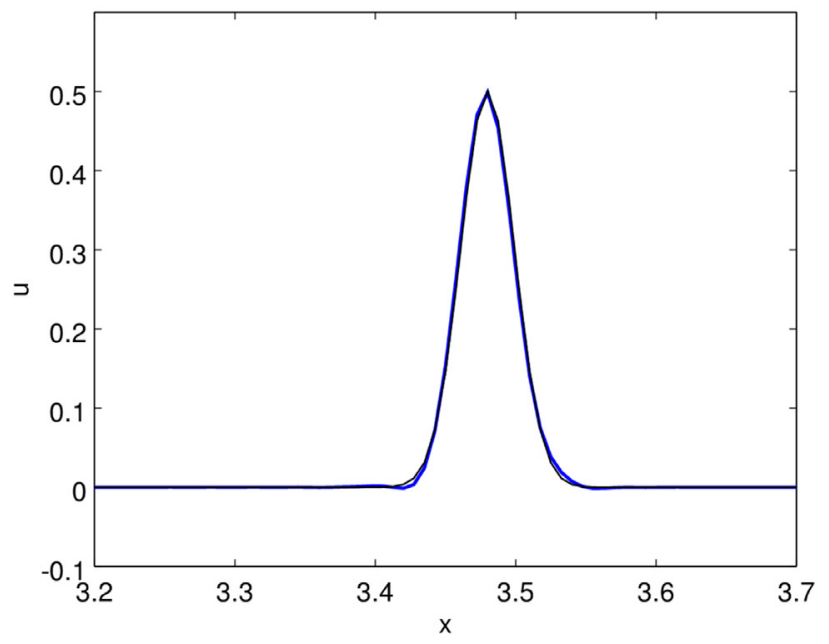

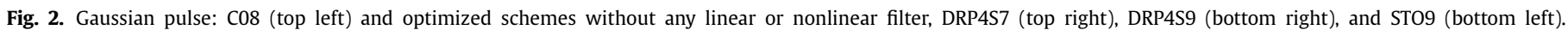

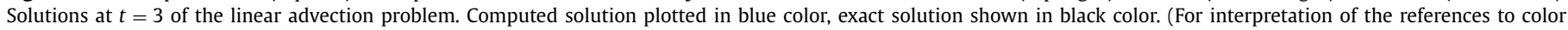
in this figure legend, the reader is referred to the web version of this article.)

real physical behavior of the wave form propagation of the initial boundary value problem (IBVP). A large percentage of DRP methods utilize least squares, $L_{1}$-norm, $L_{2}$-norm, $L_{\infty}$-norm, and other integral metrics to minimize the numerical wavenumber error over prescribed intervals in order to obtain the grid stencil coefficients. The resulting DRP schemes usually have wider grid stencils than their standard central schemes of the same order of accuracy. Low dispersive temporal discretization and special treatments for IBVPs of the different CAA applications are also needed. See Tam $[44,45]$, Brambley [6], Haras and Taasan [11], and Linders and Nordström [24], Linders et al. [25] for formulations and overviews. Some of the DRP schemes might perform poorly for decaying or growing oscillations. See Brambley [6] for a study. For discontinuous initial data and long time wave propagations of smooth acoustic waves, various space and time DRP linear filters are needed. For acoustic waves interacting with shocks and turbulence induced noise, DRP schemes with linear filters alone usually are not capable of simulating such flows.

According to Tam [44], optimized compact schemes are also DRP schemes. For over 20 years high order compact spatial discretizations in conjunction with linear high order compact filters have been methods of choice for many DNS and LES of incompressible and low speed compressible turbulent/acoustic flows due to their advantage of requiring a very low number of grid points per wavelength and flexibility in geometry handling. However, most optimized compact schemes were not designed for longtime integration and additional constraints are needed. See Haras and Taásan [11] for the construction of compact finite difference schemes for long time integration. In addition, the advantage of compact schemes seems to require additional investigation and research for compressible turbulent flows containing moderate and strong shock waves. One popular method is by employing a blending of high order compact spatial schemes with high order shockcapturing schemes. Another more efficient approach for turbulence with discontinuities is the nonlinear filter approach of Sjögreen and Yee [33], Yee and Sjögreen [49], Yee et al. [54]. They employed the high order compact scheme as their spatial base scheme. The Yee and Sjögreen studies [51] indicated that for shock-wave turbulence interactions the accuracy performance of compact schemes is similar to the central scheme of the same order under the Yee and Sjögreen nonlinear filter approach.

Objectives: Here recent progress in high order, nonlinear filter numerical method development for DNS and LES applications is reviewed. The improvement includes four major new developments: (a) Smart flow sensors were developed to replace the global ACM flow sensor $[21,22,50]$. The smart flow sensor provides the locations and the estimated strength of the necessary numerical dissipation needed at these locations and leaves the rest of the flow 

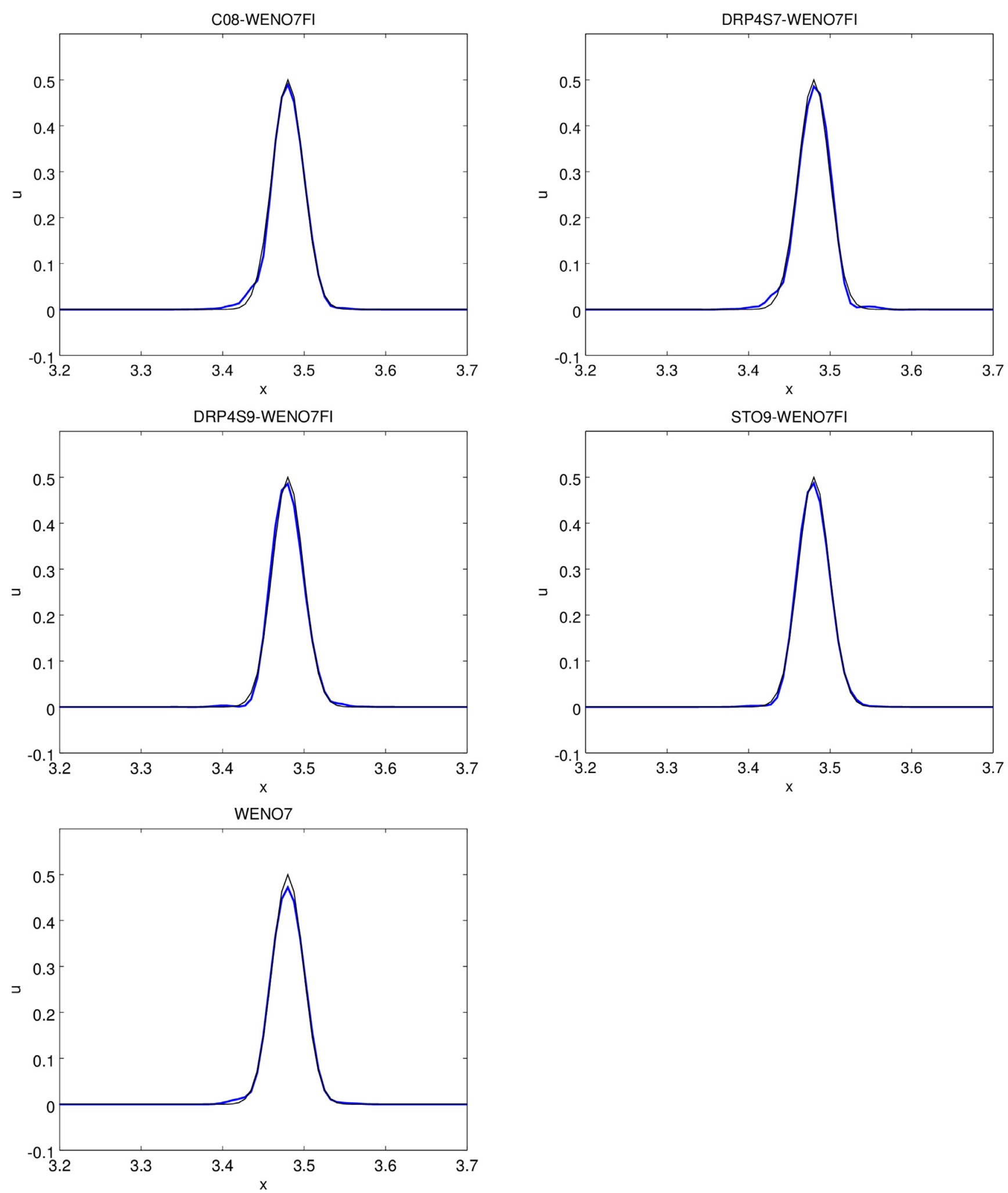

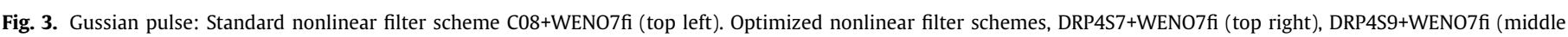

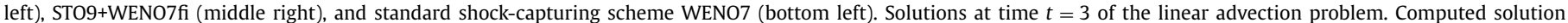

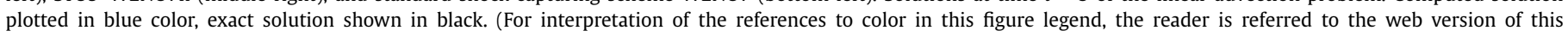
article.) 

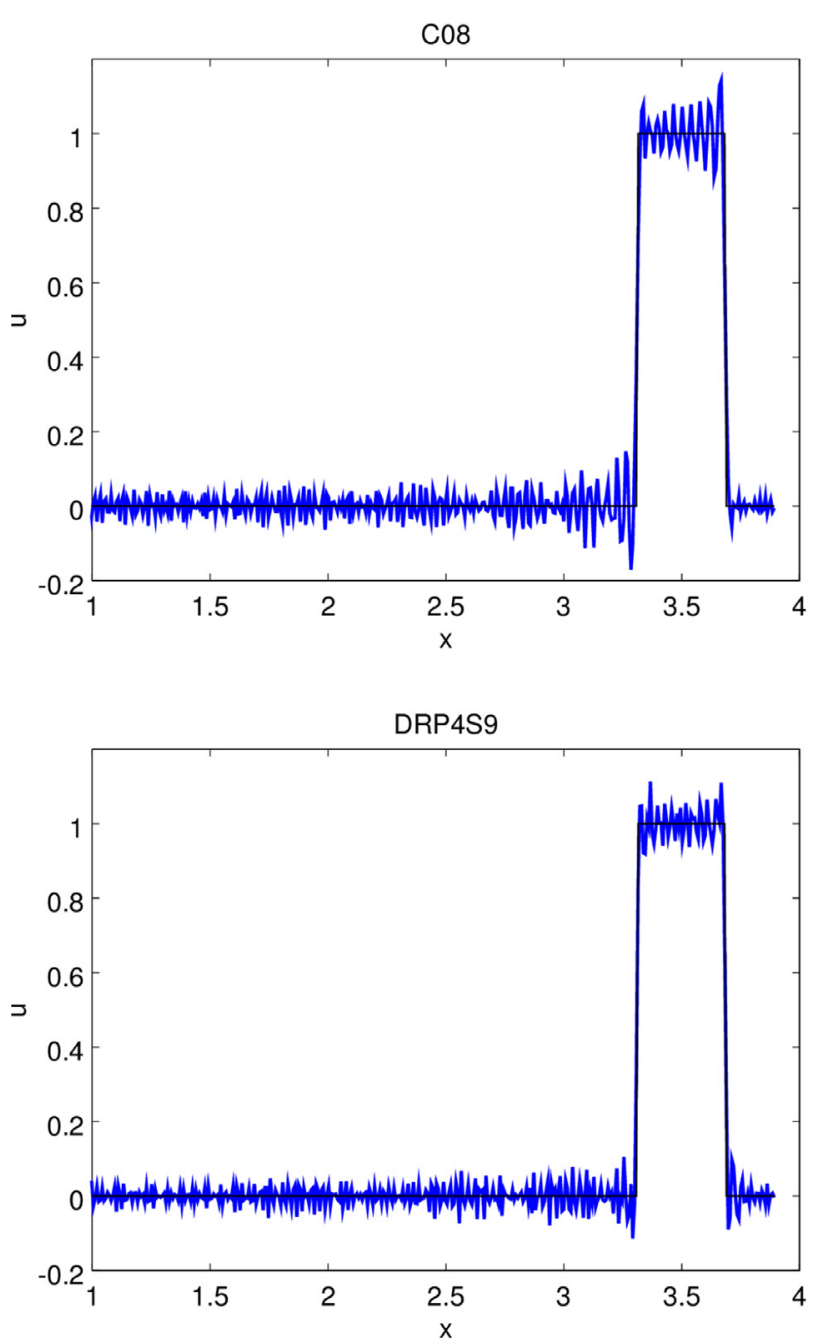

DRP4S7

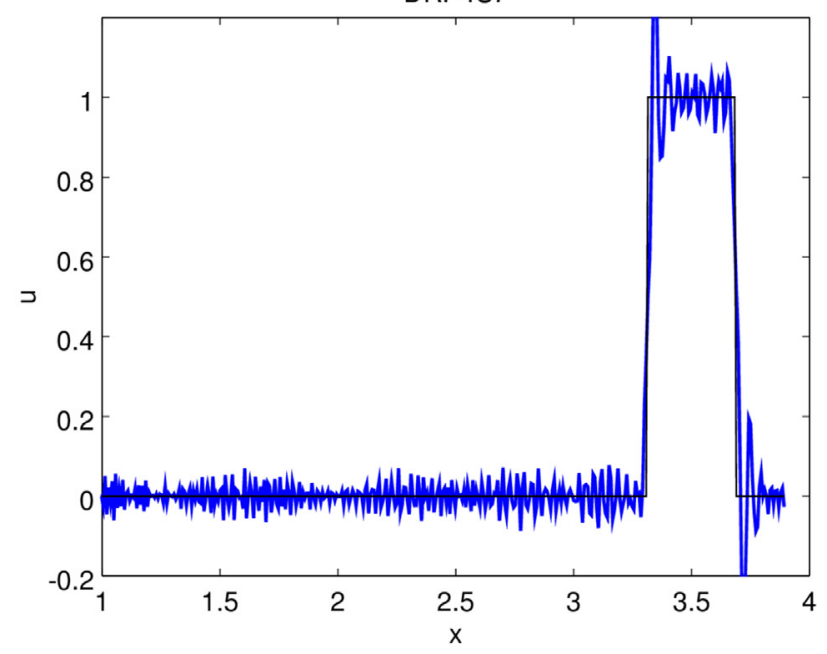

STO9

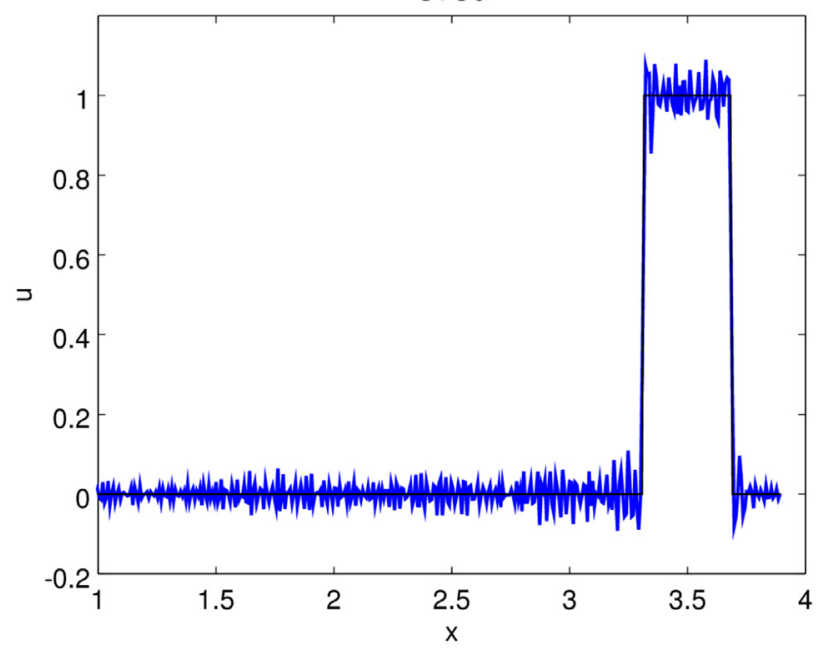

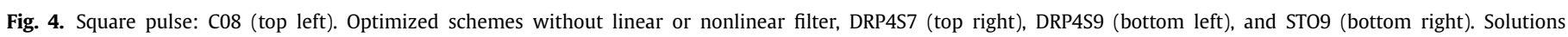

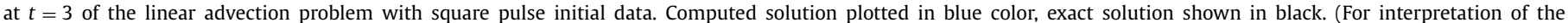
references to color in this figure legend, the reader is referred to the web version of this article.)

field free of shock-capturing dissipation. (b) Skew-symmetric splittings were developed for the compressible gas dynamics and MHD equations $[35,36]$ to improve numerical stability for long time integration. (c) High order entropy stable numerical fluxes were developed as the spatial base schemes for both the compressible gas dynamics and MHD equations [37,38]. (d) Several dispersion relationpreserving (DRP) central spatial schemes were included as spatial base schemes in the framework of our nonlinear filter scheme method approach [40].

This paper only considers several DRP central spatial schemes as the base scheme in the framework of the Yee and Sjögreen [50] low dissipative nonlinear filter method approach. DRP time discretizations are not considered. For time discretization we utilize the low dissipative fourth-order Runge-Kutta method with small time steps for the investigation to minimize dispersion error due to time discretization. The investigation is focused on the possible gain in accuracy by high order entropy numerical fluxes and DRP schemes as the base scheme over the standard central schemes of the same grid stencil for general DNS and LES compressible flow computations. As mentioned before, CAA focuses on dispersion error for long time linear wave propagation rather than the formal order of accuracy of the scheme. The resulting DRP schemes usually have wider grid stencils and an increase in CPU operations count compared to their standard central schemes of the same order of accuracy. For discontinuous initial data and long time wave propagations of smooth acoustic waves various space and time DRP linear filters are needed. For acoustic waves interacting with shocks and turbulence induced noise, DRP schemes with linear filters alone usually are not capable of simulating such flows. Due to this fact, here, the Yee and Sjögreen nonlinear filter step with shock-capturing and long time integration properties replaces the spatial DRP linear filter.

With these new scheme constructions the nonlinear filter schemes are applicable to a wider class of accurate and stable DNS and LES applications, including forced turbulence simulations where the time evolution of flows might start with low speed shock-free turbulence and develop into supersonic speeds with shocks. See $[21,22,36]$ for two of our simulations.

The next four sections give summaries of the four new major developments.

\section{An overview of skew-symmetric split approximations for gas dynamics}

Standard centered difference approximations of nonlinear conservation laws normally encounter nonlinear instabilities after a 

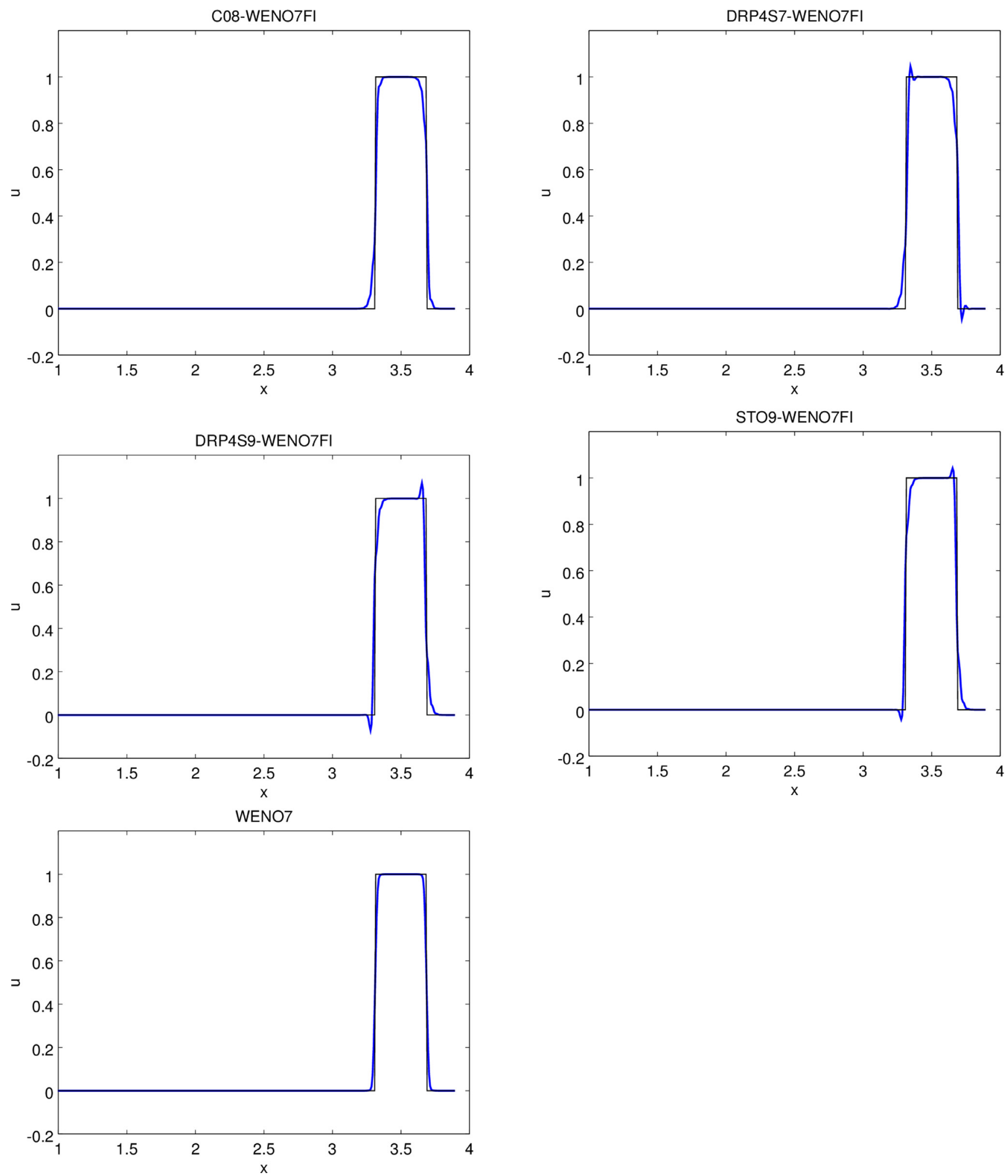

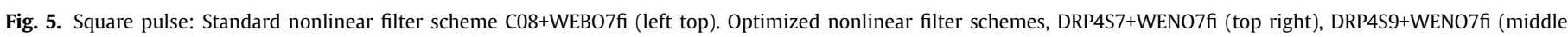

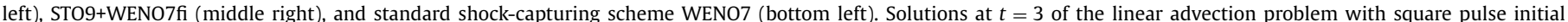

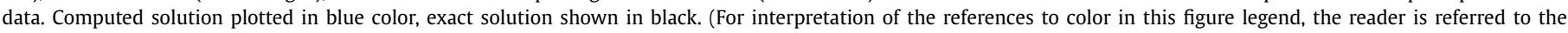
web version of this article.)

short time integration without added numerical dissipation. It is well known that the appearance of these instabilities can be delayed if the convective flux derivatives are written in an equivalent desired split form before the pure central approximation is employed. Hereafter this is referred to as a split approximation.
For example, a split approximation starts from rewriting the derivative of the product $(a b)_{x}$ as

$(a b)_{x}=\alpha(a b)_{x}+\gamma a b_{x}+\beta a_{x} b$ 

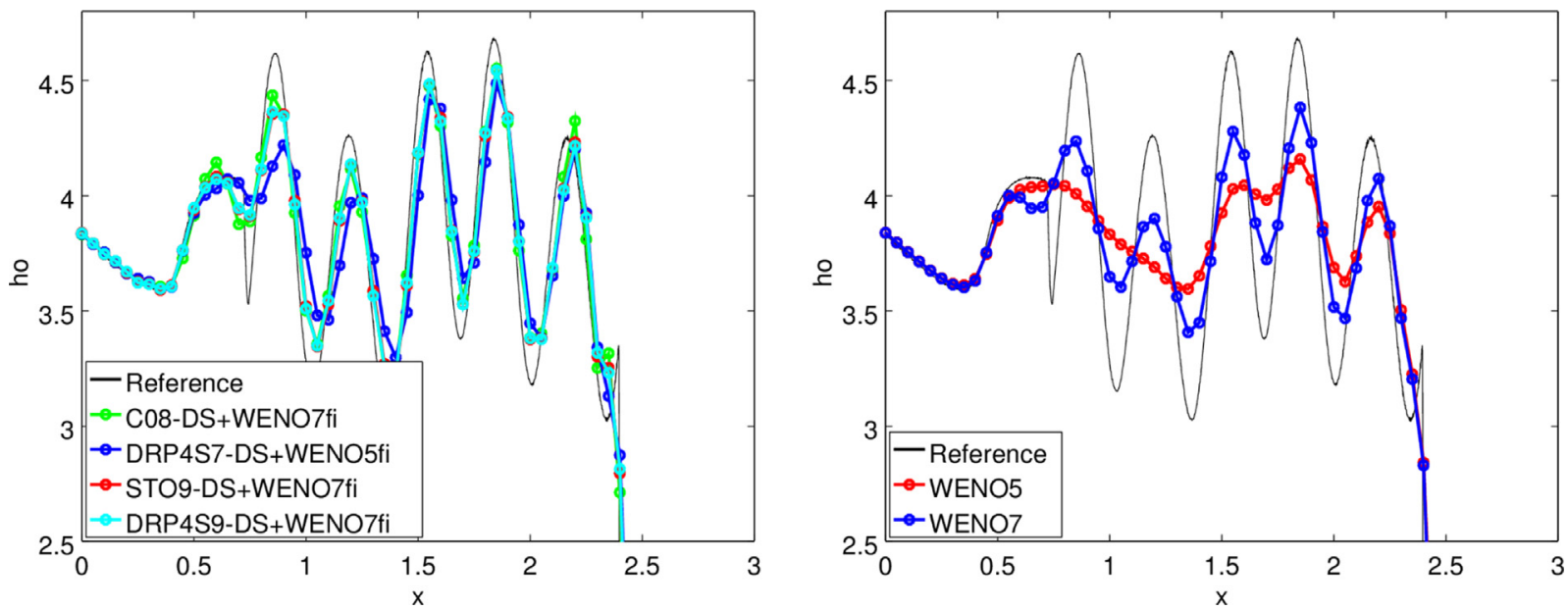

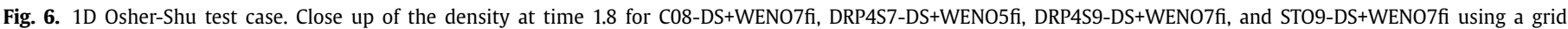
with 201 points.
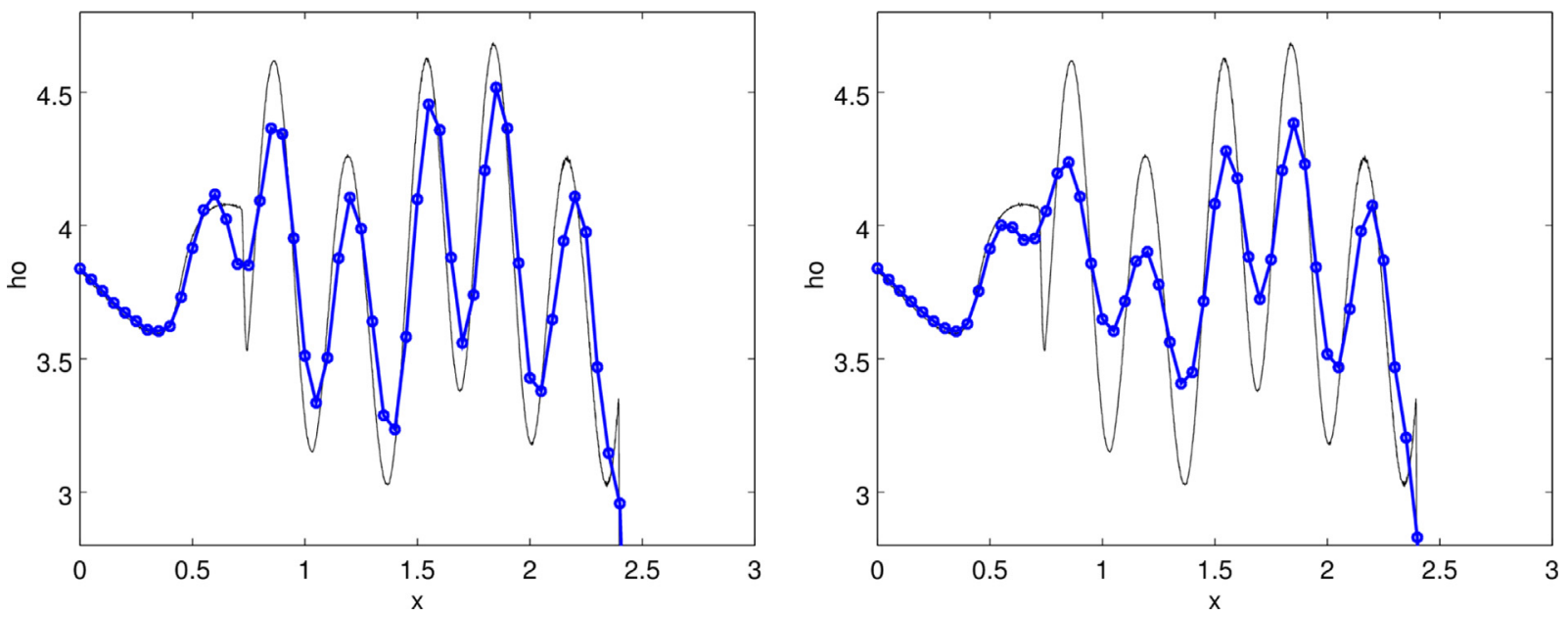

Fig. 7. 1D Osher-Shu test case: Close up of the oscillations in density at time 1.8 for C08Econs_CK+WENO7fi (left) and WENO7 (right).

before discretization. Here $a$ and $b$ are functions of $x$ and $\alpha, \gamma$ and $\beta$ are parameters so chosen to be still equivalent to the original $(a b)_{x}$ before discretization. A common split derivative is by setting $\alpha=\gamma=\beta=1 / 2$ resulting in the form

$(a b)_{x}=\frac{1}{2}(a b)_{x}+\frac{1}{2} a b_{x}+\frac{1}{2} a_{x} b$.

These methods have a long history in finite difference approximations; see, .e.g., [1,23]. See also a generalized conservative split convective derivative operators study by Pirozzoli [29]. The key mathematical idea is that formulas of type (2) can be used to estimate the $L^{2}$ norm or the energy norm of the computed solution. From physical considerations some of the splittings provide the discrete conservation of momentum or preservation of discrete kinetic energy. A well-known example is the linear system of conservation laws

$\mathbf{u}_{t}+A(x) \mathbf{u}_{x}=\mathbf{0} \quad 0<x<L$,

where $A(x)$ is a symmetric matrix, and we solve for the unknown vector $\mathbf{u}=\mathbf{u}(x, t)$ from given initial data $\mathbf{u}(x, 0)=\mathbf{u}_{0}(x)$. Boundary data are given at $x=0$ and $x=L$. To show how this is done, e.g., we rewrite (3) in an mathematically equivalent form:

$\mathbf{u}_{t}+\frac{1}{2}(A(x) \mathbf{u})_{x}+\frac{1}{2} A(x) \mathbf{u}_{x}-\frac{1}{2} A(x)_{x} \mathbf{u}=\mathbf{0}$ and define the scalar product and norm by

$(\mathbf{u}, \mathbf{v})=\int_{0}^{L} \mathbf{u}^{T} \mathbf{v} d x \quad\|\mathbf{u}\|^{2}=(\mathbf{u}, \mathbf{u})$.

A norm estimate is obtained if (4) is multiplied by $\mathbf{u}$ and integrated over $[0, L]$. We obtain

$$
\begin{aligned}
\frac{1}{2} \frac{d}{d t}\|\mathbf{u}\|^{2} & =-\frac{1}{2}\left(\mathbf{u},(A \mathbf{u})_{x}\right)-\frac{1}{2}\left(\mathbf{u}, A \mathbf{u}_{x}\right)+\frac{1}{2}\left(A_{x} \mathbf{u}, \mathbf{u}\right) \\
& =-\left.\frac{1}{2} \mathbf{u}^{T} A \mathbf{u}\right|_{0} ^{L}+\frac{1}{2}\left(A_{x} \mathbf{u}, \mathbf{u}\right),
\end{aligned}
$$

where the second equality is obtained from partial integration of $\left(\mathbf{u},(A \mathbf{u})_{X}\right)$, and from the symmetry of $A$ which allows it to be moved between the arguments of the scalar product. If the boundary data are such that $\left.\mathbf{u}^{T} A \mathbf{u}\right|_{0} ^{L} \geq 0$, then the estimate

$\frac{1}{2} \frac{d}{d t}\|\mathbf{u}\|^{2} \leq \frac{1}{2}\left(A_{x} \mathbf{u}, \mathbf{u}\right)$

holds, which under the assumption that $\max _{x}\left|A_{x}\right|$ is bounded leads to a stability estimate by use of Gronwall's lemma.

Let $x_{j}=j \Delta x, j=0, \ldots, N$ be a grid with spacing $\Delta x$, and let $\mathbf{u}_{j}(t)$ denote a numerical approximation of $\mathbf{u}\left(x_{j}, t\right)$. Consider the semi-discrete approximation of (4)

$\frac{d}{d t} \mathbf{u}_{j}+\frac{1}{2} D\left(A\left(x_{j}\right) \mathbf{u}_{j}\right)+\frac{1}{2} A\left(x_{j}\right) D \mathbf{u}_{j}-\frac{1}{2} A\left(x_{j}\right)_{x} \mathbf{u}_{j}=\mathbf{0}$, 

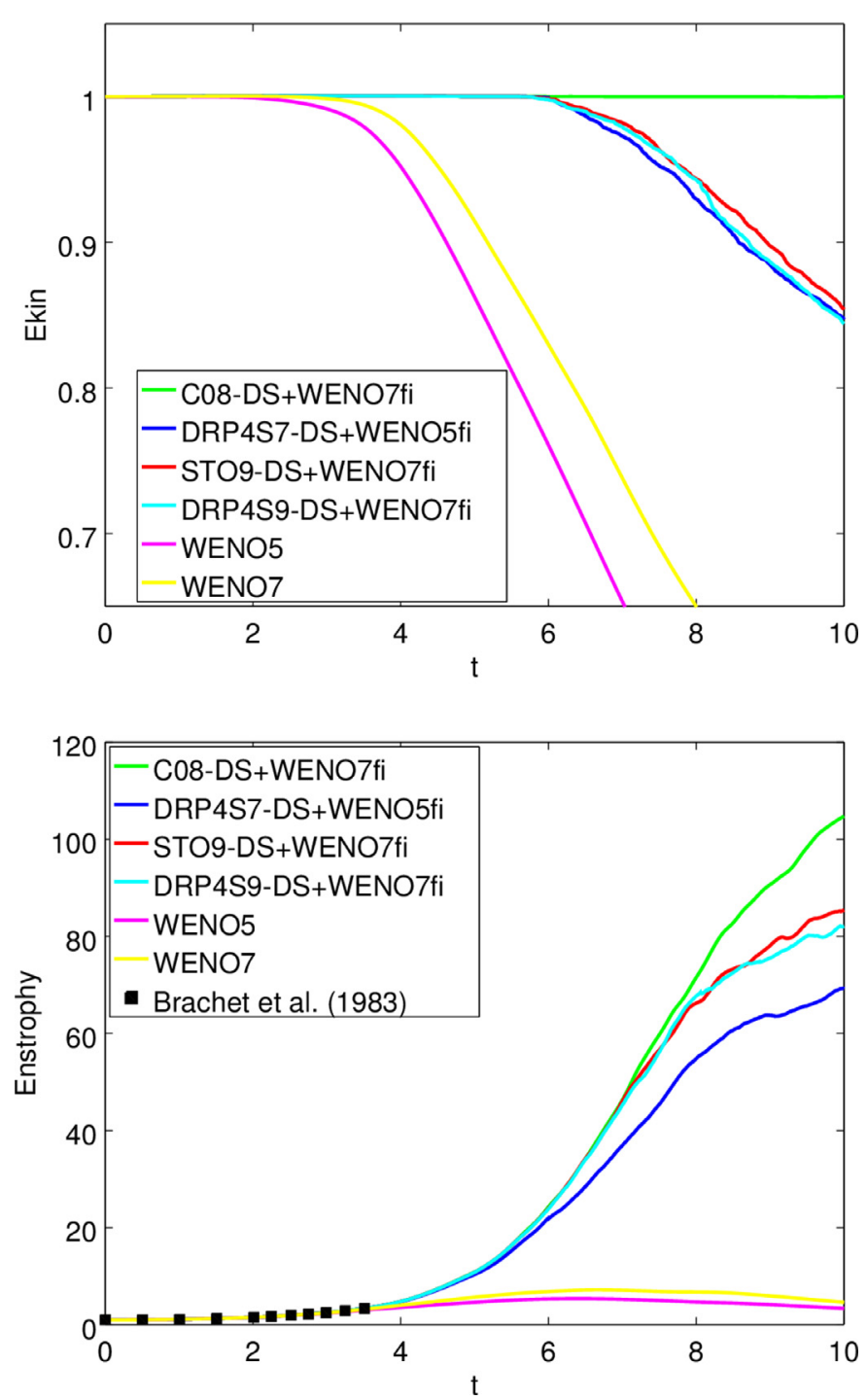

Fig. 8. 3D compressible Euler equations. Taylor-Green vortex test case. Total kinetic energy (Ekin) vs. time (top) and enstrophy vs. time (bottom) for six different methods.

where $D$ is a centered finite difference operator approximating $d / d x$. Note that $A(x)$ is a given function, so that the exact derivative $A_{x}$ can be used in (8). The discrete scalar product and norm are defined by

$(\mathbf{u}, \mathbf{v})_{h}=\sum_{j=0}^{N} \omega_{j} \mathbf{u}_{j}^{T} \mathbf{v}_{j} \Delta x \quad\|\mathbf{u}\|_{h}=(\mathbf{u}, \mathbf{u})_{h}$,

where $\omega_{j}>0$ are weights that are equal to one at most grid points, but are given special values near the boundaries $j=0$ and $j=N$. The boundary modified norm weights, together with special boundary modifications of $D$, lead to the summation-by-parts property,

$(\mathbf{u}, D \mathbf{v})_{h}=-(D \mathbf{u}, \mathbf{v})_{h}+\mathbf{u}_{N}^{T} \mathbf{v}_{N}-\mathbf{u}_{0}^{T} \mathbf{v}_{0}$,

see [39] for details. Thanks to the summation-by-parts property, the same technique that led to the estimate (7) can be used to obtain the semi discrete estimate

$\frac{1}{2} \frac{d}{d t}\|\mathbf{u}\|_{h}^{2} \leq \frac{1}{2}\left(A_{x} \mathbf{u}, \mathbf{u}\right)_{h}$.

The possible growth rate is determined by $A_{x}$ in both (7) and (9), so that the discrete estimate will have the same growth rate as the estimate of the continuous problem.
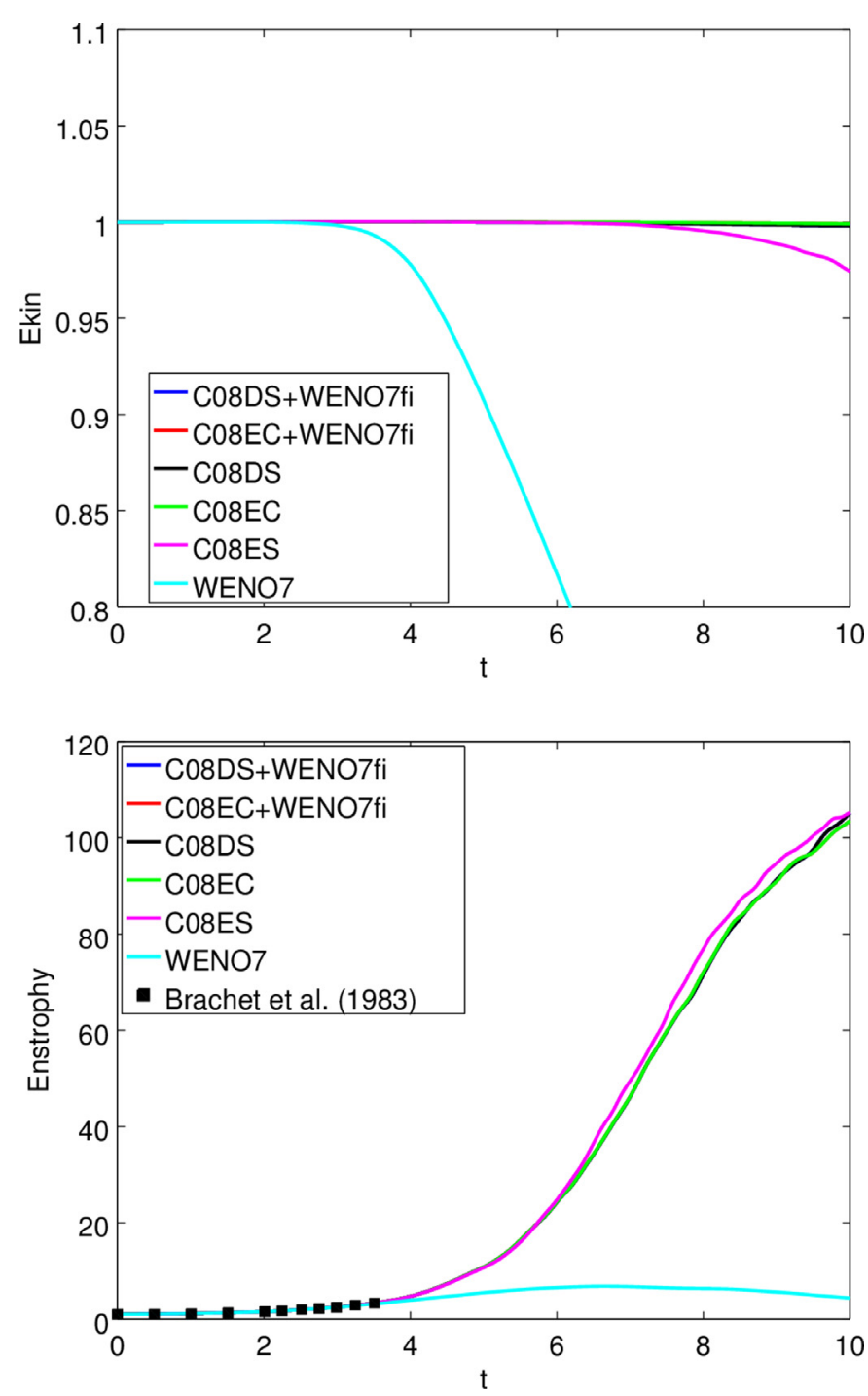

Fig. 9. 3D DNS of the Taylor-Green vortex test case. Total kinetic energy vs. time (top) and enstrophy vs. time (bottom) for six different methods.

Ducros et al.type conservative splitting: For nonlinear systems, such as the Euler equations of gas dynamics, split approximations have been used for a long time see, e.g., Ducros et al. and Blaisdel et al[2,8]..

The split approximations makes use of (2) to rewrite different terms in the Euler equations as sums of three terms. The terms of the split form (2) are approximated by

$\frac{1}{2} D(a b)+\frac{1}{2} D(a) b+\frac{1}{2} a D(b)$

where $D$ is a finite difference operator, and $a$ and $b$ are functions of $x$.

As shown in Ducros etal. [8], the approximation (10) can be written in conservation form. For example, with the second order operator $D u_{j}=\left(u_{j+1}-u_{j-1}\right) /(2 \Delta x)$, it holds that

$$
\begin{aligned}
\frac{1}{2} D(a b) & +\frac{1}{2} D(a) b \\
& +\frac{1}{2} a D(b)=\frac{1}{4 \Delta x} \Delta_{+}\left[\left(a_{j}+a_{j-1}\right)\left(b_{j}+b_{j-1}\right)\right],
\end{aligned}
$$

where $\Delta_{+} q_{j}=\left(q_{j+1}-q_{j}\right)$. 
Eq. (11) can be generalized to standard centered difference operators of $2 p$ th order of accuracy,

$D_{p} u_{j}=\frac{1}{\Delta x} \sum_{k=1}^{p} \alpha_{k}^{(p)}\left(u_{j+k}-u_{j-k}\right)$.

The coefficients $\alpha_{k}^{(p)}$ satisfy

$\sum_{k=1}^{p} k \alpha_{k}^{(p)}=\frac{1}{2} \quad \sum_{k=1}^{p} \alpha_{k}^{(p)} k^{2 n+1}=0, n=1, \ldots, p-1$.

To derive the conservative form of the split approximation for an arbitrary operator, the right hand side of the algebraic identity

$$
\begin{aligned}
a_{j+k} b_{j+k}- & a_{j-k} b_{j-k}+\left(a_{j+k}-a_{j-k}\right) b_{j}+a_{j}\left(b_{j+k}-b_{j-k}\right) \\
& =\left(a_{j+k}+a_{j}\right)\left(b_{j+k}+b_{j}\right)-\left(a_{j}+a_{j-k}\right)\left(b_{j}+b_{j-k}\right)
\end{aligned}
$$

total energy. Let $\rho_{j}, u_{j}, v_{j}, w_{j}, e_{j}$, and $p_{j}$ denote the values of the discretized variables at grid point $x_{j}$. The flux components can be written as products of two factors in many different ways, leading to different split approximations. One Ducros et al.split-type approximation of the gas dynamics flux derivative that will be used in this study is given by

$$
\left.\mathbf{f}_{x}\right|_{x=x_{j}} \approx\left(\begin{array}{l}
\frac{1}{2} D \rho_{j} u_{j}+\frac{1}{2} \rho_{j} D u_{j}+\frac{1}{2} u_{j} D \rho_{j} \\
\frac{1}{2} D \rho_{j} u_{j}^{2}+\frac{1}{2} \rho_{j} u_{j} D u_{j}+\frac{1}{2} u_{j} D \rho_{j} u_{j}+D p_{j} \\
\frac{1}{2} D \rho_{j} u_{j} v_{j}+\frac{1}{2} \rho_{j} v_{j} D u_{j}+\frac{1}{2} u_{j} D \rho_{j} v_{j} \\
\frac{1}{2} D \rho_{j} u_{j} w_{j}+\frac{1}{2} \rho_{j} w_{j} D u_{j}+\frac{1}{2} u_{j} D \rho_{j} w_{j} \\
\frac{1}{2} D u_{j}\left(e_{j}+p_{j}\right)+\frac{1}{2} u_{j} D\left(e_{j}+p_{j}\right)+ \\
\frac{1}{2}\left(e_{j}+p_{j}\right) D u_{j}
\end{array}\right),
$$

which by (17) can be written in conservative form with numerical flux function

$\mathbf{h}_{j+1 / 2}=\frac{1}{2} \sum_{k=1}^{p} \alpha_{k}^{(p)} \sum_{m=1}^{k-1}\left(\begin{array}{l}\left(\rho_{j-m}+\rho_{j+k-m}\right)\left(u_{j-m}+u_{j+k-m}\right) \\ \left(\rho_{j-m} u_{j-m}+\rho_{j+k-m} u_{j+k-m}\right)\left(u_{j-m}+u_{j+k-m}\right)+p_{j-m}+p_{j+k-m} \\ \left(\rho_{j-m} v_{j-m}+\rho_{j+k-m} v_{j+k-m}\right)\left(u_{j-m}+u_{j+k-m}\right) \\ \left(\rho_{j-m} w_{j-m}+\rho_{j+k-m} w_{j+k-m}\right)\left(u_{j-m}+u_{j+k-m}\right) \\ \left(e_{j-m}+p_{j-m}+e_{j+k-m}+p_{j+k-m}\right)\left(u_{j-m}+u_{j+k-m}\right)\end{array}\right)$.

is written in conservative form by

$$
\begin{aligned}
& \left(a_{j+k}+a_{j}\right)\left(b_{j+k}+b_{j}\right)-\left(a_{j}+a_{j-k}\right)\left(b_{j}+b_{j-k}\right) \\
& =\sum_{m=0}^{k-1}\left(a_{j-m}+a_{j+k-m}\right)\left(b_{j-m}+b_{j+k-m}\right) \\
& \quad-\sum_{m=0}^{k-1}\left(a_{j-1-m}+a_{j-1+k-m}\right)\left(b_{j-1-m}+b_{j-1+k-m}\right) .
\end{aligned}
$$

The conservative form of the split approximation becomes

$$
\begin{aligned}
\frac{1}{2} D_{p}(a b)+\frac{1}{2} D_{p}(a) b+\frac{1}{2} a D_{p}(b) \\
=\frac{1}{\Delta x} \sum_{k=1}^{p} \frac{1}{2} \alpha_{k}^{(p)}\left(\left(a_{j+k} b_{j+k}-a_{j-k} b_{j-k}\right)+a_{j}\left(b_{j+k}-b_{j-k}\right)\right. \\
\left.\quad+\left(a_{j+k}-a_{j-k}\right) b_{j}\right) \\
=\frac{1}{\Delta x} \sum_{k=1}^{p} \frac{\alpha_{k}^{(p)}}{2}\left(\sum_{m=0}^{k-1}\left(a_{j-m}+a_{j+k-m}\right)\left(b_{j-m}+b_{j+k-m}\right)\right. \\
\left.\quad-\sum_{m=0}^{k-1}\left(a_{j-1-m}+a_{j-1+k-m}\right)\left(b_{j-1-m}+b_{j-1+k-m}\right)\right) \\
=\frac{1}{\Delta x}\left(h_{j+1 / 2}-h_{j-1 / 2}\right),
\end{aligned}
$$

where the numerical flux is defined by

$h_{j+1 / 2}=\sum_{k=1}^{p} \frac{1}{2} \alpha_{k}^{(p)} \sum_{m=0}^{k-1}\left(a_{j-m}+a_{j+k-m}\right)\left(b_{j-m}+b_{j+k-m}\right)$.

To simplify the formulas of the conservative form of split approximations for systems of equations, define

$\Theta_{j+1 / 2}^{(p)}(a, b)=\sum_{k=1}^{p} \frac{1}{2} \alpha_{k}^{(p)} \sum_{m=0}^{k-1}\left(a_{j-m}+a_{j+k-m}\right)\left(b_{j-m}+b_{j+k-m}\right)$.

For the three dimensional Euler equations of gas dynamics, the $x$-direction inviscid flux is

$\mathbf{f}=\left(\rho u, \rho u^{2}+p, \rho u v, \rho u w,(e+p) u\right)^{T}$,

where $(u, v, w)$ denotes the velocities in the $x-, y$-, and $z$-directions respectively, $\rho$ denotes the density, $p$ is the pressure, and $e$ is the
The more compact notation introduced in (18) allows (21) to be rewritten as

$$
\mathbf{h}_{j+1 / 2}=\left(\begin{array}{l}
\Theta_{j+1 / 2}^{(p)}(\rho, u) \\
\Theta_{j+1 / 2}^{(p)}(\rho u, u)+\Theta_{j+1 / 2}^{(p)}(p, 1) \\
\Theta_{j+1 / 2}^{(p)}(\rho v, u) \\
\Theta_{j+1 / 2}^{(p)}(\rho w, u) \\
\Theta_{j+1 / 2}^{(p)}(e+p, u)
\end{array}\right) .
$$

A natural nonconservative splitting (not in the Ducros et al. type category):

The homogeneity property of the inviscid flux of perfect gas dynamics implies that $\mathbf{f}(\mathbf{u})=A(\mathbf{u}) \mathbf{u}$, where $A(\mathbf{u})$ is the Jacobian of $\mathbf{f}(\mathbf{u})$. To make use of the homogeneity property, a non-conservative natural splitting is

$\frac{1}{2} \mathbf{f}_{x}+\frac{1}{2} A \mathbf{u}_{x}+\frac{1}{2} A_{x} \mathbf{u}$,

where the discretization is

$\frac{d}{d t} \mathbf{u}_{j}+\frac{1}{2} D_{p} \mathbf{f}_{j}+\frac{1}{2} A_{j} D_{p} \mathbf{u}_{j}+\frac{1}{2} D_{p}\left(A_{j}\right) \mathbf{u}_{j}=0$.

Here $A_{x}$ and $D_{p} A$ denote element-wise application of differentiation and differencing respectively. The approximation (24) can be rewritten in conservative form with numerical flux

$\mathbf{h}_{j+1 / 2}=\sum_{m=1}^{5}\left(\begin{array}{c}\Theta_{j+1 / 2}^{(p)}\left(A_{1, m}, u_{m}\right) \\ \Theta_{j+1 / 2}^{(p)}\left(A_{2, m}, u_{m}\right) \\ \Theta_{j+1 / 2}^{(p)}\left(A_{3, m}, u_{m}\right) \\ \Theta_{j+1 / 2}^{(p)}\left(A_{4, m}, u_{m}\right) \\ \Theta_{j+1 / 2}^{(p)}\left(A_{5, m}, u_{m}\right)\end{array}\right)$,

where $A_{k, m}$ denotes element $(k, m)$ of the matrix-valued function $A(x)$, and $u_{m}$ denotes the $m$ th component of the vector $\mathbf{u}$.

A semi-conservative entropy splitting of the Euler flux derivatives:

Another splitting that gives entropy stability of the Euler equations of gas dynamics is by Gerritsen and Olsson [15], Olsson and Oliger [28], Yee etal. [53]. They made use of Harten's symmetrizable form of the Euler equations in terms of the entropy variables [13] to obtain a semi-discrete splitting of the Euler equations with a discrete entropy stability by the summation-by-parts approach. During the computations, the entropy splitting is written in terms 


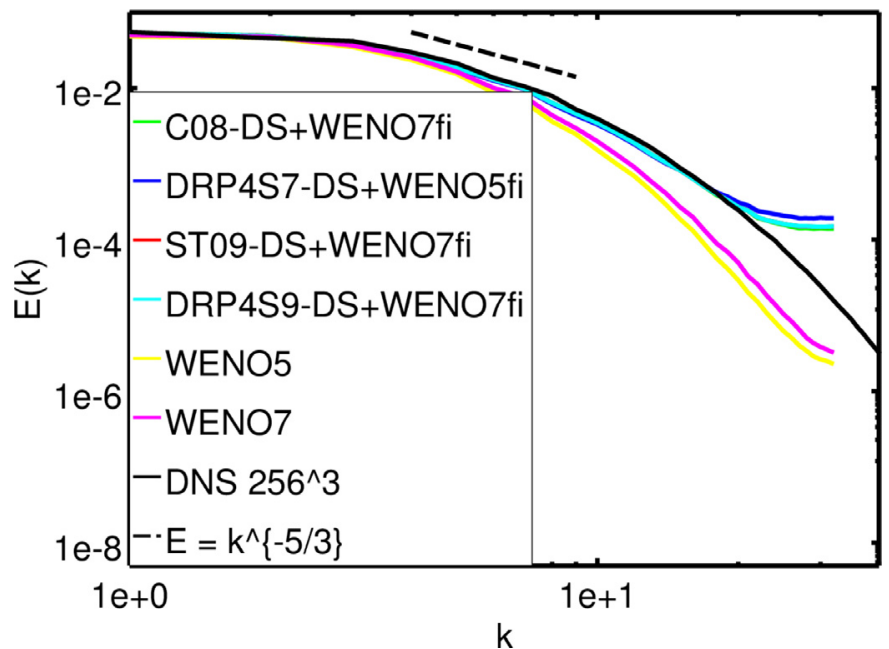

Fig. 10. 3D Isotropic turbulence test case. Energy spectra at the final time by six schemes using $64^{3}$ grid points. DNS using $256^{3}$ grid points also shown for comparison.

of the sum of a conservative portion for the interior scheme (interior grid points) and with a summation-by-parts for the boundary scheme (boundary points). Note that the Harten [13] and Gerritsen \& Olsson entropy splitting form selects the un-physical branch of the inequality and was later corrected by Yee et al. [53], hereafter referred to as the entropy splitting of the Euler equations. It is considered to be a semi-conservative splitting except at the boundary grid points. The entropy splitting of Olsson \& Oliger, Gerritsen and Olsson, and Yee etal. [15,28,53] is a splitting which is of a form that is more suitable for the discrete stable energy norm estimate technique, including boundary scheme estimate for arbitrary order of central spatial schemes. See Yee et al. [54] for the formulation. For the 1D Euler equations the inviscid flux derivative $\mathbf{f}(\mathbf{u})_{X}$ for a perfect gas is split into the following via the entropy variables $W$ discussed in Harten [13].

$\mathbf{f}_{x}=\frac{\beta}{\beta+1} \mathbf{f}_{x}+\frac{1}{\beta+1} \mathbf{f}_{W} W_{x}, \quad \beta \neq-1$

$$
\begin{aligned}
& W=\left[\begin{array}{lllll}
w_{1}, & w_{2}, & w_{3}, & w_{3}, & w_{5}
\end{array}\right]^{T} \\
& =\frac{p^{*}}{p}\left[e+\frac{\alpha-1}{\gamma-1} p, \quad-\rho u,-\rho v, \quad \rho w, \rho\right]^{T},
\end{aligned}
$$

where

$p^{*}=-\left(p \rho^{-\gamma}\right)^{\frac{1}{\alpha+\gamma}}$

and

$\beta=\frac{\alpha+\gamma}{1-\gamma}, \alpha>0$ or $\alpha<-\gamma$.

See Yee et al. [32,53,54] for the formulation, the choice for $\alpha$, and numerical examples.

Several split discretizations were compared in [14] where discretization by the entropy splitting form was shown by numerical experiments to be one of the best performing for smooth flows. For their skew-symmetric splitting extension to the ideal MHD, see Sjögreen et al. [35,36].

\section{Generalization of skew-symmetric splitting to the ideal MHD}

Due to the incomplete hyperbolic nature of the conservative ideal MHD governing equations, not all of the skew-symmetric splittings for gas dynamics can be extended to the ideal MHD. See Yee etal. [53] for a discussion. For the MHD the Ducros et al.
Table 1

Coefficients of DRP4S7, optimized over $[0,1.1]$.

\begin{tabular}{ll}
\hline$k$ & $a_{k}$ \\
\hline 1 & 0.77088238051822552 \\
2 & -0.16670590441458047 \\
3 & 0.02084314277031176 \\
\hline
\end{tabular}

[8] variants were constructed. In addition, four formulations of the MHD were considered: (a) the conservative MHD, (b) the Godunov/Powell non-conservative form, (c) the Janhunen MHD with magnetic field source terms [18], and (d) a MHD with source terms of [4]. The different formulation of the MHD equations in conjunction with the variants of Ducros etal. type skew-symmetric splitting have a strong effect on the stability of non-dissipative approximations. For their skew-symmetric splitting extension to the ideal MHD, see Yee et al., Sjögreen and Yee and Sjögreen et al. $[35,36,53]$ for the formulation. Representative test cases for both smooth flows and problems containing discontinuities for the ideal MHD can be found in $[35,36,53]$. Their results illustrate the improved stability by using the skew-symmetric splitting as part of the central base scheme instead of the pure high order central scheme.

\section{DRP schemes}

Since our objective is to utilize wavenumber optimized schemes for general DNS and LES applications, no attempt is made to obtain optimized schemes for specific IBVPs with specific initial data and boundary data. In this study three different optimized finite difference operators are considered. See Tam [44] and De Roeck et al. [31] for the development and references cited therein. These are: (a) DRP4S7, the original Tam \& Webb fourth-order accurate DRP operator with seven-point wide grid stencil, (b) DRP4S9, the fourth-order accurate DRP operator with nine-point wide grid stencil, and (c) STO9, the fourth-order accurate operator with ninepoint wide stencil by Bogey \& Bailly [3]. All three operators have antisymmetric coefficients and are optimized over wavenumber intervals $0 \leq k \Delta x \leq 1.1$ for DRP4S7 and $\pi / 16 \leq k \Delta x \leq \pi / 2$ for DRP4S9 and ST09. Here $\Delta x$ is the grid spacing and the integer $k$ is the mode number. DRP4S7 and STO9 were studied in [31].

Remark: Numerical experiments made with DRP4S7 optimized over $\pi / 16 \leq k \Delta x \leq \pi / 2$ gave worse accuracy than with DRP4S7 optimized over the more standard choice $0 \leq k \Delta x \leq 1.1$ used here. It is reasonable to expect that with fewer free parameters, the interval of optimization should be made shorter.

DRP4S7 and DRP4S9 use least square minimization of the absolute error, i.e., integral of the square of the error in wavenumber space. The STO9 scheme uses $L_{1}$ optimization of the relative error in wavenumber space, i.e., integral over the absolute value of the error divided by $k \Delta x$, since $k \Delta x$ is the exact wavenumber.

Their difference operators $D$ for the first-order derivative of a grid function $u_{j}$ are of the form

$D u_{j}=\frac{1}{\Delta x} \sum_{k=1}^{q} a_{k}\left(u_{j+k}-u_{j-k}\right)$.

Table 1 gives the coefficients of the DRP4S7 scheme, Table 2 lists the coefficients of the DRP4S9 scheme, and Table 3 shows the coefficients of the STO9 scheme. The STO9 coefficients were obtained from [31], where they are given to 12 decimals. In this work we extended the number of decimals by enforcing the fourth order accuracy constraint to high precision.

Note that the centered operators (29) are of the same asymmetric form as (12). This means that the Ducros et al. splitting de- 

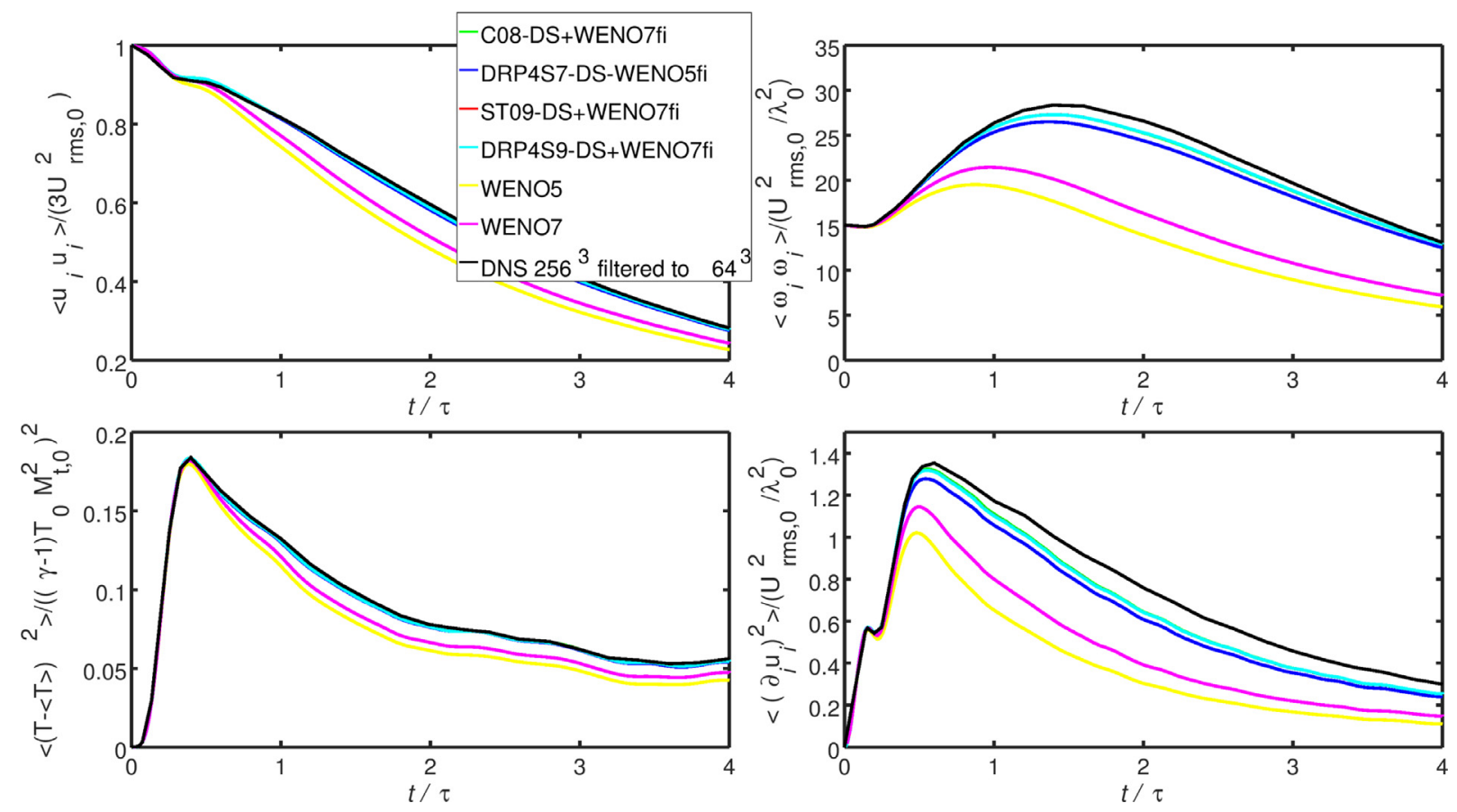

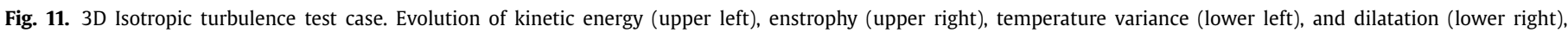
computed by six schemes, using $64^{3}$ grid points. DNS using $256^{3}$ grid points is also shown for comparison.

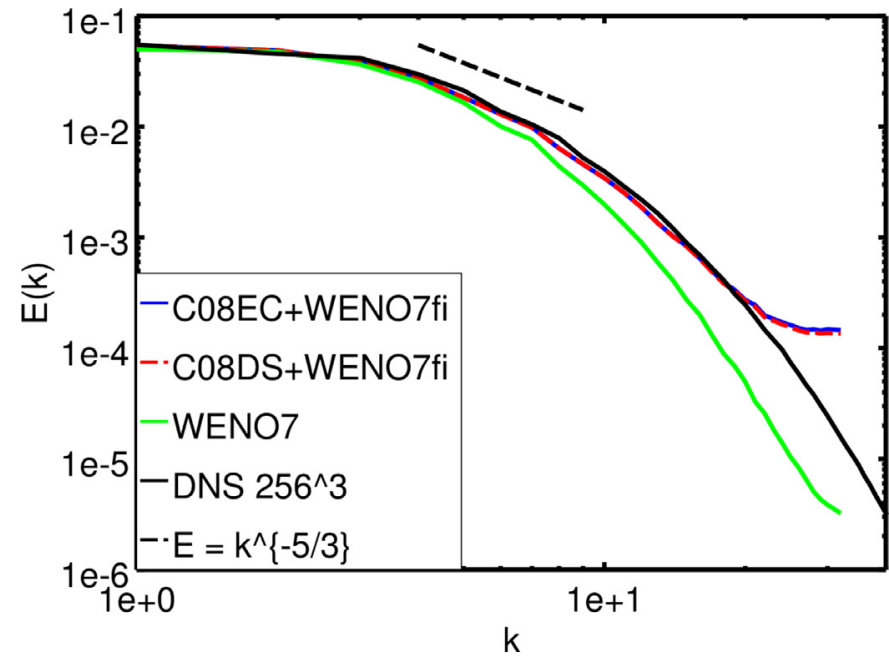

Fig. 12. 3D Isotropic turbulence test case: Energy spectra at the final time for entropy conserving base scheme (C08EC+WENO7fi, blue), Ducros split base scheme (C08DS+WENO7fi, red) and WENO7fi (green). (For interpretation of the references to color in this figure legend, the reader is referred to the web version of this article.)

\section{Table 2}

Coefficients of DRP4S9, optimized over $[\pi / 16, \pi / 2]$.

\begin{tabular}{ll}
\hline$k$ & $a_{k}$ \\
\hline 1 & 0.846863763009931 \\
2 & -0.251240526849904 \\
3 & 0.063181723773749 \\
4 & -0.008481970157843 \\
\hline
\end{tabular}

scribed in Section 2 is also straightforwardly applicable to the optimized operators described in this section. These DRP formulations are applicable to the ideal MHD equations.
Table 3

Coefficients of STO9, optimized over $[\pi / 16, \pi / 2]$, from [31].

\begin{tabular}{ll}
\hline$k$ & $a_{k}$ \\
\hline 1 & 0.841570216389881 \\
2 & -0.244678789340406 \\
3 & 0.059463699920073 \\
4 & -0.007650934367322 \\
\hline
\end{tabular}

\section{High order entropy conservative numerical fluxes}

We consider the system of conservation laws,

$\mathbf{u}_{t}+\mathbf{f}(\mathbf{u})_{x}=\mathbf{0}, \quad-\infty<x<\infty t>0$

where the unknown $\mathbf{u}=\mathbf{u}(x, t)$ is given at $t=0$. Entropy conserving schemes for (30) were introduced in the 1980s. See, e.g., [43]. These schemes are in conservation form, and admit a discrete conservation law for the entropy. An entropy, $E(\mathbf{u})$, and an entropy flux, $F(\mathbf{u})$, are two functions satisfying

$E_{\mathbf{u}}^{T} A(\mathbf{u})=F_{\mathbf{u}}^{T}$.

Here, $E_{\mathbf{u}}$ denotes the gradient of $E$ with respect to $\mathbf{u}$, and $A(\mathbf{u})$ is the Jacobian of the flux function $\mathbf{f}(\mathbf{u})$. Furthermore, $E(\mathbf{u})$ is assumed to be a convex function. The entropy variables are defined by $\mathbf{v}=$ $E_{\mathbf{u}}(\mathbf{u})$. Multiplying (30) by $\mathbf{v}^{T}$ and using

$\mathbf{v}^{T} \mathbf{u}_{t}+\mathbf{v}^{T} A \mathbf{u}_{x}=E(u)_{t}+F_{\mathbf{u}}^{T} u_{x}=E(u)_{t}+F(u)_{x}$

gives the additional conservation law for the entropy,

$E(u)_{t}+F(u)_{x}=0$.

The entropy flux potential, defined by

$\psi=\mathbf{v}^{T} \mathbf{f}-F$,

has the property that $\mathbf{f}=\psi_{\mathbf{v}}$.

If the numerical flux function $\mathbf{h}_{j+1 / 2}=\mathbf{h}\left(\mathbf{u}_{j+1}, \mathbf{u}_{j}\right)$ satisfies

$\left(\mathbf{v}_{j+1}-\mathbf{v}_{j}\right)^{T} \mathbf{h}_{j+1 / 2}=\psi_{j+1}-\psi_{j}$, 

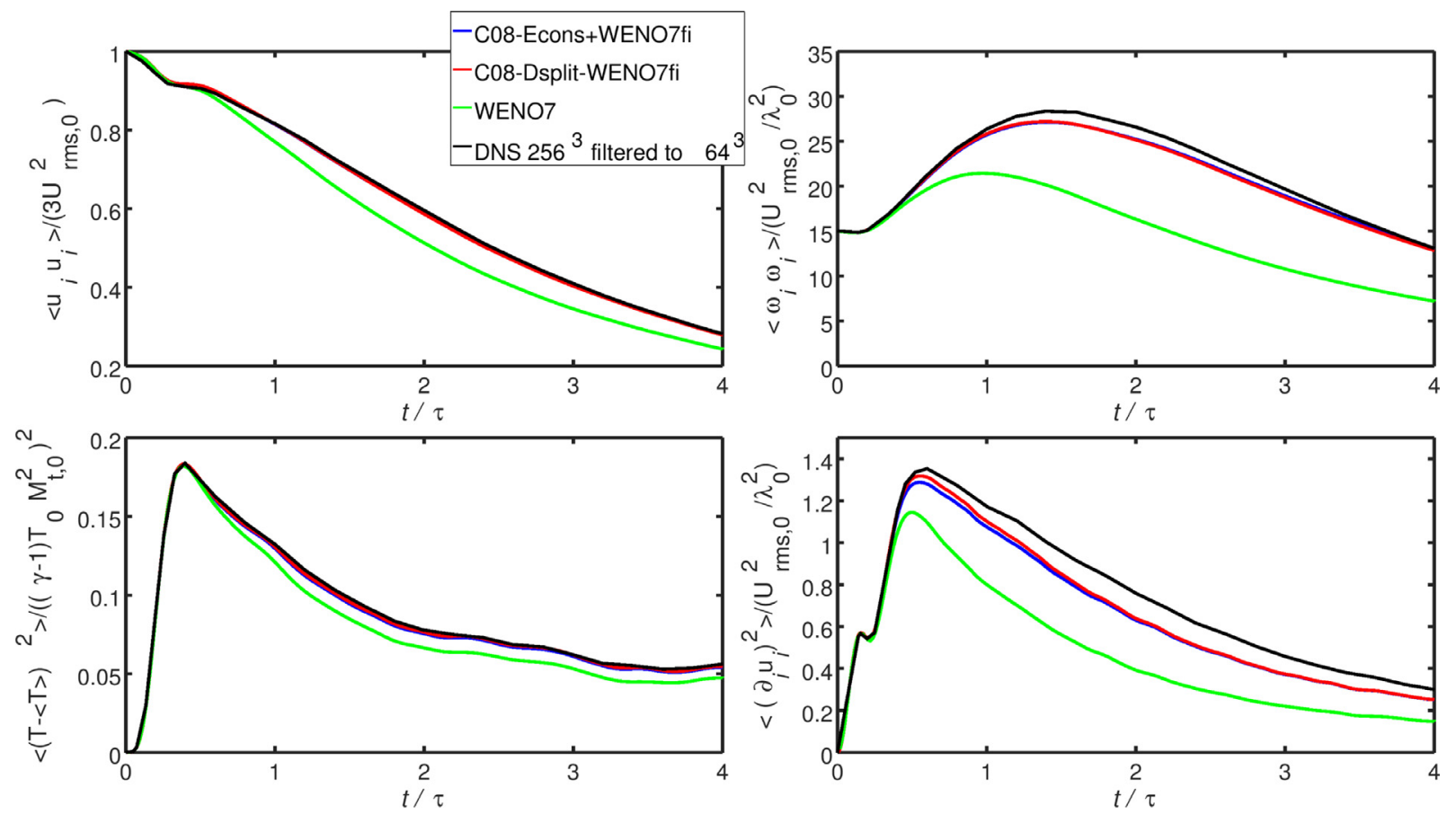

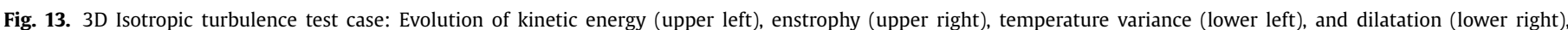

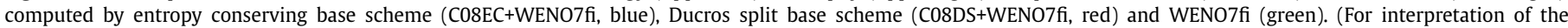
references to color in this figure legend, the reader is referred to the web version of this article.)

then the semi-discrete approximation

$\frac{d \mathbf{u}_{j}}{d t}+\frac{1}{\Delta x}\left(\mathbf{h}_{j+1 / 2}-\mathbf{h}_{j-1 / 2}\right)=\mathbf{0}$

is entropy conserving, see [42]. This result can be generalized, by defining

$\mathbf{h}_{j+k / 2}^{(k)}=\mathbf{h}\left(\mathbf{u}_{j+k}, \mathbf{u}_{j}\right), \quad k=1,2, \ldots$

where $\mathbf{h}\left(\mathbf{u}_{j+1}, \mathbf{u}_{j}\right)$ is an entropy-conserving numerical flux function. The difference scheme

$\frac{d \mathbf{u}_{j}}{d t}+\frac{1}{\Delta x} \sum_{k=1}^{p} \alpha_{k}\left(\mathbf{h}_{j+k / 2}^{(k)}-\mathbf{h}_{j-k / 2}^{(k)}\right)=\mathbf{0}$,

is then entropy conserving for arbitrary coefficients $\alpha_{k}$. It is straightforward to verify that (32) can be written in conservative form, with numerical flux function

$\mathbf{h}_{j+1 / 2}^{(e c)}=\sum_{k=1}^{p} 2 \alpha_{k} \sum_{m=1}^{k} \mathbf{h}\left(\mathbf{u}_{j+m}, \mathbf{u}_{j+m-k}\right)$.

Early entropy conserving schemes were second-order accurate. High order entropy-conserving schemes can be constructed by using the scheme (32) with suitable coefficients, $\alpha_{k}$. The $2 p$ thorder accurate standard centered finite difference operator is defined by (12) with coefficients $\alpha_{k}^{(p)}$ satisfying (13). Let $\mathbf{h}_{j+1 / 2}=$ $\mathbf{h}\left(\mathbf{u}_{j+1}, \mathbf{u}_{j}\right)$ be a second-order accurate entropy-conserving numerical flux function. The difference scheme

$\frac{d \mathbf{u}_{j}}{d t}+\frac{1}{\Delta x} \sum_{k=1}^{p} 2 \alpha_{k}^{(p)}\left(\mathbf{h}\left(\mathbf{u}_{j+k}, \mathbf{u}_{j}\right)-\mathbf{h}\left(\mathbf{u}_{j}, \mathbf{u}_{j-k}\right)\right)$,

is then 2pth-order accurate and entropy-conserving, see [34,37]. Furthermore, (33) can be written in conservative form with numerical flux function

$\mathbf{h}_{j+1 / 2}^{\text {ecp }}=\sum_{k=1}^{p} 2 \alpha_{k}^{(p)} \sum_{m=1}^{k} \mathbf{h}\left(\mathbf{u}_{j+m}, \mathbf{u}_{j+m-k}\right)$.
The scheme (33) is both $2 p$ th-order accurate and entropy conserving.

Similarly, any finite difference operator of the form (29), have an entropy-conserving counterpart for nonlinear systems of conservation laws, approximating the flux derivative $\mathbf{f}(\mathbf{u})_{x}$. For example, it is possible to define entropy conserving DRP schemes, by substituting the coefficients $\alpha_{k}^{(p)}$ in (34) for the coefficients $a_{k}$ of (29).

\section{Examples of entropy conserving numerical fluxes:}

Numerical fluxes for the 3D Euler $x$-direction flux (19). The Euler equations of compressible gas dynamics have several different entropies. The different entropies lead to different entropyconserving schemes. Furthermore, even for a fixed entropy, the entropy-conserving numerical flux function is not unique, since the entropy conservation condition (31) is only one constraint on the five (in the case of 3D Euler $x$-direction fluxes (19)) numerical flux components. Eq. (31) can be satisfied by first expressing the entropy flux potential, $\psi$ in terms of entropy variables, $\mathbf{v}$, and secondly rewrite the difference $\psi_{j+1}-\psi_{j}$ in terms of differences $\mathbf{v}_{j+1}-\mathbf{v}_{j}$. This can become algebraically involved. To simplify the algebra, a parameter vector, $\mathbf{z}$ can be introduced. The derivation is then carried out by expressing both $\psi_{j+1}-\psi_{j}$ and $\mathbf{v}_{j+1}-\mathbf{v}_{j}$ as differences $\mathbf{z}_{j+1}-\mathbf{z}_{j}$. For an example, see [48], where the derivation is expressed in detail for the equations of MHD.

This subsection will denote the average of a function $q$ by

$\{q\}=\left(q_{j+1}+q_{j}\right) / 2$

and the logarithmic average by

$q^{l n}=\frac{\ln q_{j+1}-\ln q_{j}}{q_{j+1}-q_{j}}$ 
The second-order accurate numerical flux function

$$
\mathbf{h}\left(\mathbf{u}_{j+1}, \mathbf{u}_{j}\right)=\left(\begin{array}{l}
\rho^{\ln }\{u\} \\
\rho^{\ln }\{u\}^{2}+\frac{\{\rho\}}{\{\rho / p\}} \\
\rho^{\ln }\{u\}\{v\} \\
\rho^{\ln }\{u\}\{w\} \\
h_{5}
\end{array}\right),
$$

where $h_{5}$ is the longer expression

$$
\begin{aligned}
h_{5}= & \{u\}\left(\frac{\{\rho\}}{\{\rho / p\}}+\frac{1}{\gamma-1} \frac{\rho^{l n}}{(\rho / p)^{l n}}-\frac{1}{2} \rho^{\ln }\left(\left\{u^{2}\right\}+\left\{v^{2}\right\}+\left\{w^{2}\right\}\right)\right. \\
& \left.+\rho^{\ln }\left(\{u\}^{2}+\{v\}^{2}+\{w\}^{2}\right)\right)
\end{aligned}
$$

is entropy-conserving for the entropy

$$
E=-\frac{\rho}{\gamma-1} \ln p \rho^{-\gamma}
$$

For a derivation of (35), see [38].

Another example, derived in [34], is the numerical flux function

$$
\mathbf{h}\left(\mathbf{u}_{j+1}, \mathbf{u}_{j}\right)=\left(\begin{array}{l}
\{u\}\left\{\rho(p \rho)^{-\frac{\gamma}{\gamma+1}}\right\} Q \\
\{u\}\left\{\rho u(p \rho)^{-\frac{\gamma}{\gamma+1}}\right\} Q+\left\{\frac{1}{\rho}(p \rho)^{\frac{\gamma}{\gamma+1}}\right\}\left\{(p \rho)^{\frac{1}{\gamma+1}}\right\} \\
\{u\}\left\{\rho v(p \rho)^{-\frac{\gamma}{\gamma+1}}\right\} Q \\
\{u\}\left\{\rho w(p \rho)^{-\frac{\gamma}{\gamma+1}}\right\} Q \\
\{u\}\left\{e(p \rho)^{-\frac{\gamma}{\gamma+1}}\right\} Q+\left\{\frac{u}{\rho}(p \rho)^{\frac{\gamma}{\gamma+1}}\right\}\left\{(p \rho)^{\frac{1}{\gamma+1}}\right\}
\end{array}\right),
$$

where

$$
Q=(\gamma-1) \frac{\left(p_{j+1} \rho_{j+1}\right)^{\frac{1}{\gamma+1}}-\left(p_{j} \rho_{j}\right)^{\frac{1}{\gamma+1}}}{\left(p_{j+1} \rho_{j+1}\right)^{\frac{1-\gamma}{\gamma+1}}-\left(p_{j} \rho_{j}\right)^{\frac{1-\gamma}{\gamma+1}}} .
$$

When $(p \rho)_{j+1}-(p \rho)_{j}$ is small $Q$ approaches $(p \rho)^{\frac{\gamma}{\gamma+1}}$. The numerical flux function (37) conserves the entropy

$E=\frac{1+\gamma}{1-\gamma}(\rho p)^{\frac{1}{\gamma+1}}$.

The entropy conservative high order base schemes in the numerical experiments in this paper use the numerical flux function (34) together with (35).

Entropy conservative schemes are centered non-dissipative approximations. For flows where shock waves are present, entropy conservation is unphysical and entropy conservative schemes will generate strong oscillations around discontinuities. To be useful for compressible flows, it is necessary to add some shock-capturing dissipation to the entropy conservative approximation. This is sometimes done by using linear dissipation, applied to the entropy variables. In the nonlinear filter method by Yee and Sjögreen (described in the next subsection), it is straightforward to use entropy conserving schemes. All that is needed is to substitute the centered scheme of the base scheme step by an entropy-conserving scheme.

The description above is made for the case of standard gas dynamics. Entropy conserving schemes for the equations of MHD is a subject where there has been recent progress, see, e.g., [38]. The ideas presented here, for example, the generalization of secondorder accuracy to higher order by the numerical flux (34), apply equally well to the equations of MHD.

\section{Classical central, entropy stable and DRP as base Schemes with skew-symmetric splitting as the preprocessing step in the framework of the nonlinear filter method of Yee and Sjögreen [50]}

This section gives a brief overview of the high-order nonlinear filter scheme of Yee et al. and Yee and Sjögreen [49,50,52,53] for accurate computations of DNS and LES of compressible turbulence for a wide range of flow types by introducing as little shockcapturing numerical dissipation as possible. For simplicity, the discussion uses the 3D inviscid Euler equations.

Preprocessing step by skew-symmetric splitting for gas dynamics: Before the application of a high-order non-dissipative spatial base scheme, a preprocessing step is employed to improve numerical stability. The inviscid flux derivatives of the governing equations are split in the following two ways, depending on the flow types and the desire for rigorous mathematical analysis or physical argument.

- Entropy splitting of [53] or the natural splitting described previously. These are non-conservative splittings and they are among some of the best in improving numerical stability for non-dissipative central schemes, especially for long time integration of shock-free turbulence. It has been utilized extensively in DNS of shock-free turbulence. See [32] and their later work for their wide applications.

- The Ducros etal. splitting [8] for systems (or variants of the conservative skew-symmetric splitting described earlier): These are conservative splittings and are suitable for problems with discontinuities.

Remark. For problems containing discontinuities, conservative skew-symmetric splittings should be used.

Base scheme step using the preprocessing step: A full time step is advanced using a high-order non-dissipative or very low dissipersive spatially central scheme on the split form of the governing partial differential equations (PDEs) (i.e., after the preprocessing step). For the current study, fourth-order to eighth-order classical central schemes, and the three DRP4S7, DRP4S9 and STO9 DRP schemes are considered as base schemes.

The full time step of high-order temporal discretization such as the fourth-order Runge-Kutta (RK4) method is used. It is remarked that other DRP temporal discretizations can be used for the base scheme step. See Tam [44,45], Brambley [6], and Haras and Taasan [11].

Base scheme step using the high order entropy conservative numerical fluxes The preprocessing step is left out if the spatial discretization of the base scheme is made by an entropy conserving method. In this case, again the base scheme step advances the non-dissipative discretization one full time step by an explicit time integrator.

Post-processing (Nonlinear filter step): To further improve the accuracy of the computed solution from the base scheme step, after a full time step of a non-dissipative high-order spatial base scheme on the split form of the governing equation(s), the postprocessing step is used to nonlinearly filter the solution by a dissipative portion of a high-order shock-capturing scheme with a local flow sensor. Comparable order of accuracy of the nonlinear filter dissipation with the base scheme usually is considered. For nonentropy satisfying shock-capturing schemes it is assumed that entropy satisfying fixes for both 1D and multi-D are employed [55]. For extreme flows positivity-preserving shock-capturing schemes should be used. See Kotov etal. $[19,20]$ for some performance of positivity-preserving nonlinear filter schemes.

The flow sensor provides locations and amounts of built-in shock-capturing dissipation that can be further reduced or eliminated. At each grid point a local flow sensor is employed to analyze the regularity of the computed flow data. Only the strong discontinuity locations would receive the full amount of shockcapturing dissipation. In smooth regions no shock-capturing dissipation would be added unless high frequency oscillations develop, owning to the possibility of numerical instability in long time integrations of nonlinear governing PDEs. In regions with strong turbu- 
lence, if needed, a small fraction of the shock-capturing dissipation would be added to improve stability.

Note that the filter numerical fluxes only involve the inviscid flux derivatives, regardless if the flow is viscous or inviscid. If viscous terms are present, a matching high order central difference operator (as the inviscid difference operator) is included on the base scheme step. For ease of summation-by-parts numerical boundary closure implementation for the viscous flux derivatives, the same inviscid central difference operator for the first derivative is employed twice for the viscous flux derivatives.

Remark. For the gas dynamics the post-processing (nonlinear filter step) is employed for all of the equation set for both non-reacting and reacting flows. For the MHD on a uniform Cartesian grid, in order to obtain zero discrete divB error without any divB cleaning, the nonlinear filter step is not employed for the three magnetic field equations. See Yee and Sjögreen [52] for details.

For simplicity of presentation, consider the 3D Euler equations

$\frac{\partial U}{\partial t}+\frac{\partial E}{\partial x}+\frac{\partial F}{\partial y}+\frac{\partial G}{\partial z}=0$,

where $E, F$ and $G$ are inviscid fluxes in the $x, y$ and $z$ directions, respectively.

Let $U^{*}$ be the solution after the completion of the full time step of the base scheme step. The final update of the solution after the filter step is

$$
\begin{aligned}
U_{j, k, l}^{n+1}= & U_{j, k, l}^{*}-\frac{\Delta t}{\Delta x}\left[H_{j+1 / 2, k, l}^{*(x)}-H_{j-1 / 2, k, l}^{*(x)}\right] \\
& -\frac{\Delta t}{\Delta y}\left[H_{j, k+1 / 2, l}^{*(y)}-H_{j, k-1 / 2, l}^{*(y)}\right]-\frac{\Delta t}{\Delta z}\left[H_{j, k, l+1 / 2}^{*(z)}-H_{j, k, l-1 / 2}^{*(z)}\right],
\end{aligned}
$$

$H_{j+1 / 2, k, l}^{*(x)}$ and $H_{j-1 / 2, k, l}^{*(x)}$ are "filter" numerical fluxes in the $x$ direction in terms of Roe's average states based on $U^{*}$. Similarly $H_{j, k+1 / 2, l}^{*(y)}$ and $H_{j, k, l+1 / 2}^{*(z)}$ are numerical filter fluxes in the $y$ - and $z$-directions respectively. From here on, the simplified notation $H_{j+1 / 2}^{*}$ will be used for the $x$-direction filter flux $H_{j+1 / 2, k, l}^{*(x)}$, and the grid point indices $k, l$ will be suppressed on all quantities defined below. The discussion will focus on the $x$-direction flux, the $y$ - and $z$-direction fluxes are defined similarly. The filter flux is defined in characteristics components by

$H_{j+1 / 2}^{*}=R_{j+1 / 2} \bar{H}_{j+1 / 2}$,

where $R_{j+1 / 2, k, l}$ is the matrix of right eigenvectors of the Jacobian of the inviscid flux vector in terms of Roe's average states based on $U^{*}$. Denote the elements of the filter numerical flux vector $\bar{H}_{j+1 / 2, k, l}$ by $\bar{h}_{j+1 / 2}^{l}, l=1,2, \ldots, 5$, where $\bar{h}_{j+1 / 2}^{l}$ has the form

$\bar{h}_{j+1 / 2}^{l}=\frac{\kappa_{j+1 / 2}^{l}}{2} w_{j+1 / 2}^{l} \phi_{j+1 / 2}^{l}$.

Here $w_{j+1 / 2}^{l}$ is a flow sensor to activate the nonlinear numerical dissipation portion of a high order shock-capturing scheme $\frac{1}{2} \phi_{j+1 / 2}^{l}$, and $\kappa_{j+1 / 2}^{l}$ is a positive flow dependent parameter that is less than or equal to one to control the amount of shock-capturing dissipation to be used. The nonlinear dissipative portion of a highresolution shock-capturing scheme " $\frac{1}{2} \phi_{j+1 / 2}^{l}$ " can be any shockcapturing scheme. The choice of the parameter $\kappa_{j+1 / 2}^{l}$ can be different for different flow types and is automatically chosen by using the local $\kappa_{j+1 / 2}^{l}$ described in [50]. The flow sensor $w_{j+1 / 2}^{l}$ can be a variety of formulae introduced in the literature or can be switched from one flow sensor to another, depending on the computed flow data at that particular location. For a variety of local flow sensors with automatic selection of the proper parameter, depending on different flow type, see [50]. The form of Tauber-Sandham
[47] for the filter numerical flux uses the Ducros et al. flow sensor [9] as $\kappa_{j+1 / 2}^{l}$ and the Harten artificial compression method formula (ACM) [12] as the flow sensor indicated in [54] and similarly in [26] is part of the Yee and Sjögreen adaptive numerical dissipation control generalization filter formulae. For the numerical experiments presented, we mainly concentrate on the wavelet flow sensor of Yee and Sjögreen, the Ducros et al. flow sensor [9] and the artificial compression method flow sensor of [54]. For the wavelets and ACM flow sensors, see the aforementioned references cited. The Ducros et al. flow sensor was designed mainly to capture flows containing shocks and vorticity with the divcurl tolerance of the form:

$s w=\frac{(\nabla \cdot \mathbf{u})^{2}}{(\nabla \cdot \mathbf{u})^{2}+\omega^{2}+\varepsilon}$.

Here $\mathbf{u}$ is the velocity vector, $\omega$ is the vorticity magnitude and $\varepsilon$ is a small number to avoid division by zero (e.g., $10^{-6}$ ). The Ducros etal. flow sensor consists of a cut off parameter $\delta$ as an input parameter based on the value of $s w$ that can be used to switch on or off the dissipative portion of the high order shock-capturing scheme. If $\delta$ is set to be one, the dissipation only switches on when it encounters a shock wave. For a lower value of the cut off $\delta$ parameter, vorticity can be detected. The $\delta$ parameter is used as the $\kappa_{j+1 / 2}^{l}$ for the Durcros et al. flow sensor.

The low Mach number $\kappa$ curve was developed in Yee and Sjögreen [50] and detail is omitted here. Local flow sensors for a wide spectrum of flow speed and shock strength developed in $[21,22,50]$ are also omitted here.

The aforementioned high order nonlinear filter method is valid for the four forms of the MHD formulation and the four skewsymmetric splittings of the MHD to be used as the preprocessing step. In addition, the aforementioned high order nonlinear filter method is valid for the four forms of the MHD formulation and the different high order entropy conservative numerical fluxes such as the spatial base schemes discussed in Sections 4 and 5 of Sjögreen and Yee $[37,38]$.

From here on, without loss of generality, the term "a split scheme" refers to the use of a high order central scheme to discretize a skew-symmetric splitting form of the inviscid flux derivatives. If the three considered DRP4S7, DRP4S9 and STO9 schemes are used as the base schemes, and the dissipative portion of the seventh-order WENO (WENO7) is used as the nonlinear filter, they are denoted by DRP4S7+WENO7fi, DRP4S9+WENO7fi, and STO9+WENO7fi respectively. Similarly if WENO5fi is used, they are denoted by DRP4S7+WENO5fi, DRP4S9+WENO5fi, and STO9+WENO5fi. If an eighth-order classical central difference operator is used as the base scheme for the aforementioned three DRP schemes, it is denoted by C08+WENO7fi. If Ducros et al. splitting is used, e.g., it is denoted by C08-DS+WENO7fi.

Note that any good high-resolution high order shock-capturing methods are suitable as the dissipative portion of the nonlinear filter approach. Here standard Jiang and Shu [17] WENO5 and WENO7 are chosen for the numerical experiments. Optimized WENO schemes are not as robust for our nonlinear filter approach.

\section{Numerical results}

This section shows some numerical results for compressible gas dynamics. Extensive grid refinement and scheme comparison, including 3D forced turbulence, LES and MHD simulations can be found by the authors and collaborators in [21,22,35-38] and references cited therein. The test cases shown here include problems with smooth flows, problems containing shock waves, shock-free turbulence and turbulence with weak shocks. These test cases are well known test cases in the literature and will be used to illustrate the performance of the proposed methods. The first two test 
cases are commonly used simple test cases as a prelude to turbulent computations. The comparison results for a $2 \mathrm{D}$ compressible Euler simulation of isentropic vortex convection can be found in the aforementioned references. The last two test cases are 3D DNS computations of the Taylor-Green vortex and isotropic turbulence. They are included to show that our proposed schemes are suitable for DNS of turbulent flows.

Here, for illustration purposes, only two smart flow sensors (among the many variants indicated in [50] and Kotov et al. [21,22]) are chosen for the numerical experiment for the nonlinear filter approach. Except for the DNS test cases, the third-order B-spline wavelet flow sensor developed in Sjögreen and Yee [33] was employed. For the DNS computations the Ducros et al. flow sensor was employed. This is due to the fact that the Ducros et al. flow sensor is most suited for these two particular DNS computations. See Kotov et al. [21,22] for the DNS and LES of Navier-Stokes computations using the nonlinear filter method, including a supersonic stationary shock interacting with turbulent initial data.

\subsection{Scalar linear wave results}

In this subsection the scalar advection equation

$u_{t}+u_{x}=0 \quad t \geq 0$

is solved on an interval $0 \leq x \leq 3.9$ with periodic boundary conditions. Initial data will be either a Gaussian pulse or a square pulse. The domain and initial data are scaled such that the problem is equivalent to the linear advection problem solved in [31]. It is noted that Ducros etal. splitting is not applicable to linear constant coefficient equations. In addition, the Ducros et al. flow sensor is only applicable to higher than 1D nonlinear Euler/NavierStokes equations.

\subsubsection{Smooth initial data: Gaussian pulse}

The advection Eq. (43) is solved with initial data $u(x, 0)=\frac{1}{2} e^{-K\left(x-x_{c}\right)^{2}}$,

where $K=1369.2$ and $x_{c}=0.48$. The spatial discretization has 520 grid points, the CFL number is 0.1 , and the problem is solved to time $t=3$, which since the wave speed is 1 , means that the pulse has traveled 3 length units. Fig. 1 shows a close up of the initial data near $x_{c}$. The pulse is resolved with approximately 15 grid points. Computed results are displayed in Figs. 2 and 3. The ninepoint stencil optimized nonlinear filter schemes, DRP4S9+WENO7fi and STO9+WENO7fi, appear to be more accurate than the other methods, especially on the lower left side of the pulse. The dissipative nature of the WENO7 scheme is also visible as a somewhat lower peak value than the other methods.

\subsubsection{Discontinuous initial data: Square pulse}

The advection Eq. (43) is solved with initial data

$u(x, 0)= \begin{cases}1 & 0.3124 \leq x<0.6875 \\ 0 & \text { otherwise }\end{cases}$

Also for this initial data, the spatial discretization has 520 grid points, the CFL number is 0.1 , and the problem is solved to time 3 . Computed results are displayed in Figs. 4 and 5. The C08, DRP4S7, DRP4S9 and STO9 without nonlinear filter exhibit oscillatory solutions. With linear filters and DRP time discretization indicated in $[31,44]$, the oscillations are suppressed. See [31] for the result. Here, the nonlinear filter version of the DRP methods are able to suppress some of the oscillations. However, the WENO7 scheme, which is designed for discontinuous solutions, shows the best performance. The optimized nine-point stencil nonlinear filter methods agree somewhat better with the exact solution, especially near the 'corners' of the pulse, than DRP4S7+WENO7fi and C08+WENO7fi.

\subsection{D compressible euler test case with shocks: Shu-Osher problem}

The Shu-Osher problem [41] is a one-dimensional Mach 3 shock moving into an oscillatory density. A highly oscillatory flow field (1D turbulent flow) develops behind the shock wave. The problem is defined for the one dimensional Euler equations with $\gamma=1.4$ and initial data

$(\rho, u, p)= \begin{cases}(3.857143,2.629369,10.33333), & x<-4 \\ (1+0.2 \sin 5 x, 0,1), & x \geq 4\end{cases}$

on the domain $-5 \leq x \leq 5$. The grid has 201 points, corresponding to discretization size $\Delta x=0.05$. The CFL number was 0.3 for all computations in this subsection. The nonlinear numerical dissipation is multiplied with sensors designed to activate it only in the neighborhood of shocks. In the computations shown here a wavelet sensor was used with two wavelet levels and a cut-off smoothness exponent 0.5 .

The left subplot of Fig. 6 shows the density at the final time computed by the optimized stencil schemes DRP4S7, DRP4S9, and STO9, implemented in the Ducros et al.split form of the equations. The seventh order WENO dissipation is used as postprocessing filter (DRP4S7-DS+WENO7fi, DRP4S9-DS+WENO7fi, STO9DS+WENO7fi). Also shown in the figure is the solution by the standard centered eighth-order nine-point scheme, with Ducros et al.splitting and WENO7 filter (C08-DS+WENO7fi). The computed densities by STO9-DS+WENO7fi and DRP4S9-DS+WENO7fi are almost on top of each other. STO9-DS+WENO7fi, plotted in red, is almost completely covered by the cyan colored DRP4S9DS+WENO7fi.

For comparison, the right subplot of Fig. 6 shows the solution by the Jiang and Shu WENO5 and WENO7 schemes. Except for DRP4S7-DS+WENO5fi, the filter scheme captures the physical oscillations well on this very coarse grid. Accuracy compares very favorably with the results from the WENO7 scheme. Higher accuracy can be obtained with a local smart flow sensor in the use of the Yee and Sjögreen nonlinear filter scheme. Here we only show results using one global flow sensor for the computation. Results for the same problem, but using entropy conserving base schemes instead of split schemes together with the WENO7 filter denoted by C08Econs_CK+WENO7fi is shown in Fig. 7. The accuracy is similar to C08-DS+WENO7fi. One advantage of split schemes is their computational cost is in general lower than the cost of entropy conserving schemes.

Fig. 7 shows a close up of the oscillatory regions of the plots in Fig. 6.

\subsection{D compressible Euler shock-free turbulence test case: Taylor-Green vortex}

The Taylor-Green vortex [46] is a well-known shock-free compressible turbulence test problem that has been studied extensively. Extensive scheme comparison is reported in Kotov et al. [21] for DNS and LES simulations with grid refinement studies employing the high order central nonlinear filter scheme using the Ducros et al. splitting. The 3D Euler equations of compressible gas dynamics are solved with $\gamma=5 / 3$. The computational domain is a cube with sides of length $2 \pi$ and with periodic boundary conditions in all three directions. The initial data are

$\rho=1, \quad p=100+\{(\cos (2 z)+2)(\cos (2 x)+\cos (2 y))-2\} / 16$,

$u=\sin x \cos y \cos z, v=-\cos x \sin y \cos z, w=0$.

The problem is solved to time 10 on a uniform grid with $64^{3}$ grid points. A CFL number of 1.4 was used. 
The total kinetic energy of the exact solution is constant in time. Fig. 8 shows the evolution of the total kinetic energy for the four different nonlinear filter schemes. All four methods conserve the kinetic energy extremely well. As the flow evolves, smaller scales are created, which causes an increase in the enstrophy. The enstropy increase for the three different DRP nonlinear filtered schemes can be seen in the bottom subplot of Fig. 8. These computed results agree well with the filtered DNS using a $256^{3}$ grid reported in [22] and the Brachet etal. [5] linearized theory (up to time $t<4$ ). The results from WENO5 and WENO7, which perform poorly, are also included for comparison. C08-DS-WENO7fi performs the best.

Fig. 9 shows the evolution of the total kinetic energy for the three different schemes, the tenth-order central scheme with Ducros etal. splitting in conjunction with WENO7fi C08DS+WENO7fi (blue), the seventh-order entropy conserving nonlinear filter scheme C08EC+WENO7fi (red), and the reference C10DS (tenth-order central scheme with Ducros etal. splitting, black). Results by C08DS (eighth-order central with Ducros et al. splitting), C08EC (eighth-order entropy conservative numerical flux), C08ES (eighth-order central with Yee et al. entropy splitting [53]), and WENO7 are included for comparison. All methods preserve the kinetic energy extremely well. As the flow evolves, smaller scales are created, which causes an increase in the enstrophy. The enstropy increase for the three different schemes can be seen in the bottom subplot of Fig. 9. Closer inspection shows that C08EC+WENO7fi is closer to the reference solution, C10DS, than C08DS+WENO7fi after time $t=9$. These computed results agree well with the filter DNS using a $256^{3}$ grid reported in [22] and the Brachet et al. [5] linearized theory (up to time $t<4$ ). The figures are from Sjögreen etal. [36] where slightly different notations were used.

Remark. For this nearly incompressible low speed test case the schemes of choice in the literature are spectral and high order compact or central schemes with summation-by-parts boundary closures in conjunction with their respective high order linear filters. The nonlinear filter step is not needed. This study is to show the versatility of the proposed approach when a priori knowledge of the flow structure is not known, and/or for flows with a time varying random forcing and a wide range of flow speed regimes during the entire time-accurate evolution. See the Appendix of Kotov et al. for an illustration [22] or Sjögreen and Yee [36].

\subsection{D compressible Euler turbulence with shocklets test case: isotropic turbulence with eddy shocklets}

This test case is a decaying compressible isotropic turbulence with eddy shocklets. For high enough turbulent RMS Mach numbers, weak shocks (shocklets) develop from the turbulent motion. In this test the initial turbulent Mach number is 0.6. The equations are solved using $\gamma=1.4$. The computational domain is a cube with side length $2 \pi$ and with periodic boundary conditions in all three directions. The initial datum is a random divergence free velocity field, $u_{i, 0}, i=1,2,3$, that satisfies

$\frac{3}{2} u_{R M S, 0}^{2}=\frac{1}{2}\left\langle u_{i, 0}, u_{i, 0}\right\rangle=\int_{0}^{\infty} E(k) d k$

with energy spectrum

$E(k) \sim k^{4} e^{-2\left(k / k_{0}\right)^{2}}$.

The computations below were made with $u_{R M S, 0}=1$ and $k_{0}=4$. The angular brackets denote averaging over the entire computational domain. The density and pressure fields are constant initially. See [21] for definitions of the quantities and more details about the set up of the problem. The simulation is run to the final time 4, using CFL number 1.4 .
Fig. 10 compares the energy spectra computed using four nonlinear filter methods. Spectra from WENO5 and WENO7 are also shown. Fig. 11 shows the evolution in time of kinetic energy, enstrophy, temperature variation, and dilatation for the same schemes. The notation on the $y$-axis in Fig. 11 uses the angular brackets to denote volume average,

$\langle q\rangle=\frac{1}{(2 \pi)^{3}} \int_{0}^{2 \pi} \int_{0}^{2 \pi} \int_{0}^{2 \pi} q(x, y, z) d x d y d z$.

and the summation convention is used with velocity vector $\left(u_{1}\right.$, $\left.u_{2}, u_{3}\right)$ so that the upper left subplot shows $\frac{1}{3}\left\langle u^{2}+v^{2}+w^{2}\right\rangle$, normalized by $u_{R M S, 0}^{2}=1$. The upper right subplot shows the enstrophy averaged over the volume, $\left\langle\omega_{i} \omega_{i}\right\rangle$, where $\omega_{i}, i=1,2,3$ are the components of the velocity curl vector. The plotted enstrophy is normalized by $u_{R M S, 0}^{2} / \lambda_{0}^{2}$, where $\lambda_{0}$ is the Taylor microscale of the initial data. For this computation $\lambda_{0}=1 / 2$. The lower left subplot shows the square of the RMS temperature, $\left\langle(T-<T>)^{2}\right\rangle$ normalized by $\left((\gamma-1) T_{0} M_{t, 0}^{2}\right)^{2}$, where the initial temperature $T_{0}=1$ and $M_{t, 0}$ denotes the initial turbulent Mach number. Finally, the lower right subplot shows the average velocity divergence, $\left\langle\left(u_{x}+\right.\right.$ $\left.\left.v_{y}+w_{z}\right)^{2}\right\rangle$ normalized by $u_{R M S, 0}^{2} / \lambda_{0}^{2}$.

In Figs. 10-11, the results with C08+WENO7fi, STO9+WENO7fi, and DRP4S9+WENOfi are indistinguishable, and the cyan colored curve (which was plotted last) covers the green and red curves. The results show agreement between the central base scheme and the optimized (DRP, STO) base schemes. These computed results agree well with the filtered DNS using a $256^{3}$ grid reported in [22]. Performance of DNS and LES by WENO5 and WENO7 using the same $64^{3}$ coarse grid is also reported in [22]. WENO5 and WENO7 results are more diffusive than the results obtained by nonlinear filter methods. Fig. 12 shows the energy spectra comparing the seventh-order entropy conserving base scheme C08ECWENO7fi (blue) with a computation from the seventh-order Ducros et al. split base scheme C08DS+WENO7fi (red-). The difference between the two computations is very small. Fig. 13 shows the evolution in time of kinetic energy, enstrophy, temperature variation, and dilatation for the two base schemes. The results show agreement between the two schemes. These computed results agree well with the filtered DNS using a $256^{3}$ grid reported in [22]. Performance of WENO5 and WENO7 using the same $64^{3}$ coarse grid is also reported in [22]. WENO5 and WENO7 results are more diffusive than the result shown here.

\section{Conclusions}

An overview of the Yee and Sjögreen and Kotov et al. [21,22,3538,50 ] high order numerical methods for compressible flows has been presented for long time wave propagation of smooth flows, DNS of shock-free turbulence, and DNS of turbulence with weak, moderate and strong shocks, including forced turbulent flows. This work combines four key ingredients to improve stability and accuracy of DNS computations. Although LES results are not shown, this improvement carries over to LES simulations for the subject flows. The four ingredients are: (a) Smart flow sensors were developed to replace the global ACM flow sensor [21,22,50]. The smart flow sensor provides the locations and the estimated strength of the necessary numerical dissipation needed at these locations and leaves the rest of the flow field free of shock-capturing dissipation. (b) Skewsymmetric splitting of the inviscid flux derivative as a preprocessing step before the application of the spatial base scheme, (c) high order entropy stable conservative numerical fluxes as the spatial base scheme, (d) DRP centered schemes as the spatial base scheme, and replacing various high order linear filters by the dissipative portion of high order high-resolution shock-capturing scheme with smart flow sensor to minimize spurious high frequency oscillation and Gibbs phenomena across discontinuities. These methods are 
evaluated on standard test problems in compressible fluid dynamics, Taylor-Green vortex, shock/turbulence interaction, and isotropic turbulence with shock waves.

Among all of the numerical experiments studied in [21,22,35$38,50]$, only selected four test cases are shown here. Numerical experiments demonstrate that DRP schemes and standard central schemes of the same grid stencil width in the framework of the Yee \& Sjögreen nonlinear filter approach are of similar accuracy as long as the grid resolution is not extremely high. Their CPU operations count for the same grid stencil width is the same per method evaluation. The high order entropy stable conservative numerical fluxes under the Yee \& Sjögreen nonlinear filter approach have almost identical accuracy as the central schemes of the same order employing the skew-symmetric splitting. However, the CPU operations count associated with of the high order entropy stable conservative numerical fluxes is among the highest of the three approaches. Due to the fact that our coding of the entropy stable conservative numerical fluxes is not optimized for parallel computing in the same way for the other two approaches, the efficiency measure among the three approaches cannot be shown here until such optimizations are done.

\section{Acknowledgement}

Financial support from the NASA TTT/RCA program for the second author is gratefully acknowledged. The authors are grateful to Dr. Alan Wray of NASA Ames Research Center for the numerous valuable discussion throughout the course of this work.

\section{References}

[1] Arakawa A. Computational design for long-term numerical integration of the equations of fluid motion: two-dimensional incompressible flow. Part I J Comput Phys 1966;1:119-43.

[2] Blaisdell GA, Spyropoulos ET, Qin JH. The effect of the formulation of nonlinear terms on aliasing errors in spectral methods. Appl Num Math 1996;21:207-19.

[3] Bogey C, Bailly C. A family of low dispersive and low dissipative explicit exicit schemes for computing the aerodynamic noise. In: AIAA-paper 2002-2509, 8th ARAA/CEAS Aeroacoustics Conference \& Exhibit, June 17-19; 2002. Breckenridge Colorado.

[4] Brackbill JU, Barnes DC. The effect of nonzero $\nabla \cdot \mathbf{b}$ on the numerical solution of the magnetohydrodynamics equations. J Comput Phys 1980;35:426-30.

[5] Brachet M, Meiron D, Orszag S, Nickel B, Morf R, Frisch U. J Fluid Mech $1983 ; 130: 411$

[6] Brambley EJ. Optimized finite-difference (DRP) schemes perform poorly for decaying or growing oscillations. J Comput Phys 2015;324:258-74.

[7] Chandrashekar P, Klingenberg C. Entropy stable finite volume scheme for ideal compressible MHD on 2-d cartesian meshes. SIAM J Numer Anal 2016;54:1313-40.

[8] Ducros F, Laporte F, Soulères T, Guinot V, Moinat P, Caruelle B. High-order fluxes for conservative skew-symmetric-like schemes in structured meshes: application to compressible flows. J Comput Phys 2000;161:114-39.

[9] Ducros F, Ferrand V, Nicoud F, Weber C, Darracq D, Gacherieu C, et al. Large-eddy simulation of the shock/turbulence interaction. J Comput Phys 1999;152:517-49.

[10] Fu L, Hu X, Adams N. A family of high-order targeted ENO scheme for compressible-fluid simulations. J Comput Phys 2016;305:333-59.

[11] Haras Z, Taásan S. Finite difference schemes for long time integrations. J Comput Phys 1994;114:265.

[12] Harten A. The artificial compression method for computations of shocks and contact discontinuities: III. self-adjusting hybrid schemes. J Comput Phys 1978;32:363-89.

[13] Harten A. On the symmetric form of systems for conservation laws with entropy. J Comput Phys 1983;49:151.

[14] Honein AE, Moin P. Numerical aspects of compressible turbulence simulations. Stanford University, Report no. TF-92, Dept of Mech Eng; 2005.

[15] Gerritsen M, Olsson P. Designing an efficient solution strategy for fluid flows. I. A stable high order finite difference scheme and sharp shock resolution for the euler equations. J Comput Phys 1996;129:245.

[16] Guo Y, Xiong T, Shi Y. A positivity-preserving high order finite volume compact-WENO scheme for compressible euler equations. J Comput Phys 2014;274:505. doi:10.1016/j.jcp.2014.06.046.

[17] Jiang CS, Shu CW. Efficient implementation of weighted ENO schemes. J Comput Phys 1996;128:202.
[18] Janhunen P. A positive conservative method for MHD based on HLL and roe methods. J Comput Phys 2000;160:649-61.

[19] Kotov DV, Yee HC, Sjögreen B. Comparative study of high order positivity-preserving WENO schemes. In: Proceedings of the ICCFD8, Chengdu, Sichuan, China, July 14-18; 2014.

[20] Kotov DV, Yee HC, Panesi M, Prabhu DK, Wray AA. Computational challenges for simulations related to the NASA electric arc shock tube (EAST) experiments. J Comput Phys 2014;269:215-33.

[21] Kotov DV, Yee HC, Wray AA, Sjögreen B, Kritsuk AG. Numerical disipation control in high order shock-capturing schemes for LES of low speed flows. J Comput Phys 2016;307:189-202.

[22] Kotov DV, Yee HC, Wray AA, Sjögreen B. High order numerical methods for dynamic SGS model of turbulent flows with shocks. Commun Comput Phys 2016;19:273-300.

[23] Kreiss HO. Numerical methods for solving time-dependent problems for partial differential equations. In: Les Presses de l'Université de Montreéal; 1978.

[24] Linders K, Nordström J. Uniformly best wavenumber approximations by spatial central difference operators. J Comput Phys 2015;300:695-709.

[25] Linders K, Kupiainen M, Nordström J. Summation-by-parts operators with minimal dispersion error for accurate and efficient flow calculations. In: Proceedings of the AIAA SciTech Conference, Jan. 4-8; 2016. San Diego.

[26] Lo SC, Blasisdell G, Lyrintzis A. High order shock capturing schemes for turbulence calculations. J Num Meth Fluids 2010;62:473.

[27] Martin MP, Taylor EM, Wu M, Weirs VG. A bandwidth-optimixed WENO scheme for effective direct numerical simulations of compressible turbulence. J Comput Phys 2006;220:270-89.

[28] Olsson P, Oliger J. Energy and maximum norm estimates for nonlinear conservation laws. RIACS Technical Report 94.01; 1994.

[29] Pirozzoli S. Generalized conservative approximations of split convective derivative operators. J Comput Phys 2010;219:7180-90.

[30] Pirozzoli S. Numerical methods for high-speed flows. Annu Rev Fluid Mech 2011;43:163-94. doi:10.1146/annurev-fluid-122109-160718.

[31] De Roeck W, Desmet W, Baelmans M, Sas P. An overview of high-order finite difference schemes for computational aeroacoustics. In: Proceedings of ISMA2004, September 20-22; 2004. Katholieke Universiteit Leuven, Belgium.

[32] Sandham ND, Li Q Yee HC. Entropy splitting for high-order numerical simulation of compressible turbulence. J Comput Phys 2002;23:307-22.

[33] Sjögreen B, Yee HC. Multiresolution wavelet based adaptive numerical dissipation control for high order methods. J Scientific Computing 2004;20:211-55.

[34] Sjögreen B, Yee HC. On skew-symmetric splitting and entropy conservation schemes for the euler equations. In: Proceedings of of ENUMATH09, June 29July 2; 2009. Uppsala University, Sweden.

[35] Sjögreen B, Yee HC. Skew-symmetric splitting and stability of high order central schemes. In: To appear: proceeding of ASTRONUM-2016, June 6-10; 2016. Monterey, CA, USA

[36] Sjögreen B, Yee HC. Improving numerical stability via skew-symmetric splitting in multiscale gas dynamics and MHD turbulence flows. In: Extended version of the proceeding of ASTRONUM-2016, June 6-10; 2016. Monterey, CA, USA, submitted to J. Comput. Phys., 2016

[37] Sjögreen B, Yee HC. On high order entropy conserving numerical flux for multiscale gas dynamics and MHD turbulent simulations. In: Proceeding of ICOSAHOM-2016, June 27-July 1; 2016. Rio de Janeiro, Brazil

[38] Sjögreen B, Yee HC. Construction of high order entropy conserving numerical flux for multiscale gas dynamics and MHD turbulent simulations. In: Extended version of the proceeding of ICOSAHOM-2016, June 27-July 1; 2016. Rio de Janeiro, Brazil, submitted to J. Comput. Phys., 2016

[39] Strand B. Summation by parts for finite difference approximations for $d / d x$. J Comput Phys 1994;110:47-67.

[40] Sjögreen B, Yee HC. Accuracy consideration by DRP schemes for DNS and LES of compressible flow computations. In: Special issue in computers \& fluids in honor of Prof. Toro's 70th birthday; 2016. Submitted.

[41] Shu C-W, Osher SJ. Efficient implementation of essentially non-oscillatory shock capturing schemes. J Comput Phys 1989;83:32-78.

[42] Tadmor E. Numerical viscosity and the entropy condition for conservative difference schemes. Math Comput 1984;43:369-81.

[43] Tadmor E. Numerical viscosity of entropy stable schemes for systems of conservation laws. I Math Comput 1987;49:91-103.

[44] Tam CKW. A CAA primer for practicing engineers. AEDC-TR-08-2, april. Arnold engineering development center, Arnold air force base, Tennssee; 2008.

[45] Tam CKW. Computational aeroacoustics: a wave number approach. Cambridge aerospace series, vol.33. New York: Cambridge University Press; 2012.

[46] Taylor G, Green A. Proc R Soc Lond A 1937;158:499.

[47] Tauber E, Sandham ND. Comparison of three large-eddy simulatitons of shock-induced turbulent separation bubbles. Shock Waves 2009;19:469-78.

[48] Winters AR, Gassner GJ. Affordable, entropy conserving and entropy stable flux functions for the ideal MHD equations. J Comput Phys 2016;304:72-108.

[49] Yee HC, Sjögreen B. Development of low dissipative high order filter schemes for multiscale navier-stokes MHD systems. J Comput Phys 2007;225:910-34.

[50] Yee HC, Sjögreen B. High order filter methods for wide range of compressible flow speeds. In: Proceedings of the ICOSAHOM09, June 22-26; 2009. Trondheim, Norway

[51] Yee HC, Sjögreen B. Adaptive filtering and limiting in compact high order methods for multiscale gas dynamics and MHD systems. Comput Fluids 2008;37:593-619. 
H.C. Yee, B. Sjögreen/Computers and Fluids 000 (2017) 1-18

[52] Yee HC, Sjögreen B. Efficient low dissipative high order schemes for multiscale MHD flows, II: minimization of $\nabla \cdot b$ numerical error. J Sci Comput 2006;29:115-64.

[53] Yee HC, Vinokur M, Djomehri MJ. Entropy splitting and numerical dissipation. J Comp Phys 2000;162:33-81.
[54] Yee HC, Sandham ND, Djomehri MJ. Low-dissipative high order shock-capturing methods using characteristtic-based filters. J Comput Phys 1999:150:199-238.

[55] Yee HC, Klopfer GH, Montangne JL. High-reolution shock-capturing schemes for inviscid and viscous hypersonic flows. J Comput Phys 1990;88:31-61. 